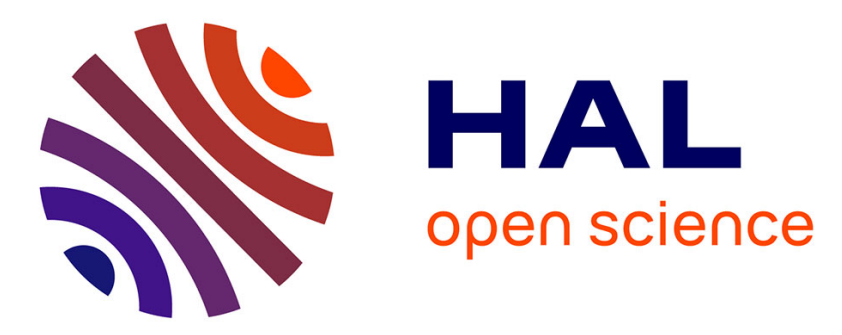

\title{
Harmonic spin-orbit angular momentum cascade in nonlinear optical crystals
}

Yutao Tang, Kingfai Li, Xuecai Zhang, Junhong Deng, Guixin Li, Etienne Brasselet

\section{- To cite this version:}

Yutao Tang, Kingfai Li, Xuecai Zhang, Junhong Deng, Guixin Li, et al.. Harmonic spin-orbit angular momentum cascade in nonlinear optical crystals. Nature Photonics, 2020, 14 (11), pp.658-662. 10.1038/s41566-020-0691-0 . hal-03126750

\section{HAL Id: hal-03126750 https://hal.science/hal-03126750}

Submitted on 1 Dec 2021

HAL is a multi-disciplinary open access archive for the deposit and dissemination of scientific research documents, whether they are published or not. The documents may come from teaching and research institutions in France or abroad, or from public or private research centers.
L'archive ouverte pluridisciplinaire HAL, est destinée au dépôt et à la diffusion de documents scientifiques de niveau recherche, publiés ou non, émanant des établissements d'enseignement et de recherche français ou étrangers, des laboratoires publics ou privés. 


\title{
Harmonic spin-orbit angular momentum cascade in nonlinear optical crystals
}

\author{
Yutao Tang ${ }^{1,4}$, Kingfai Li $^{1,4}$, Xuecai Zhang, Junhong Deng ${ }^{1,2}$, Guixin Li ${ }^{1,2} \bowtie$ and Etienne Brasselet $\oplus^{3 凶}$
}

\begin{abstract}
Optical angular momentum-based photonic technologies demonstrate the key role of the optical spin-orbit interaction that usually refers to linear optical processes in spatially engineered optical materials'. Re-examining the basics of nonlinear optics of homogeneous crystals under circularly polarized light ${ }^{2,3}$, we report experiments on the enrichment of the spinorbit angular momentum spectrum of paraxial light. The demonstration is made within the framework of second-harmonic generation using a crystal with three-fold rotational symmetry. Four spin-orbit optical states for the second harmonic field are predicted from a single fundamental state owing to the interplay between linear spin-orbit coupling and nonlinear wave mixing; three of these states are experimentally verified. Besides representing a spin-controlled nonlinear route to orbital angular multiplexing ${ }^{4}$, modal vortex light sources ${ }^{5,6}$, high-dimensional parametric processes ${ }^{7}$ and multi-state optical magnetization ${ }^{8}$, our findings suggest that the fundamentals of nonlinear optics are worth revisiting through the prism of the spin-orbit interaction of light.
\end{abstract}

Almost three decades after optical beams with helical phase profiles were identified as carrying a well-defined orbital angular momentum (OAM) per photon ${ }^{9}$, efforts to generate and control single or multiple OAM states are increasing ${ }^{10}$. This trend is nurtured by the advent of spin-controlled photonics, which sets the polarization state of light as an independent degree of freedom with which to tailor the optical spatial degrees of freedom ${ }^{1}$. The development of technologies enabling spin-controlled OAM states started in the 2000s in the framework of linear optics with the emergence of devices made of (1) homogeneous anisotropic materials ${ }^{11,12}$, (2) structured isotropic materials ${ }^{13}$ and (3) structured anisotropic materials ${ }^{14}$. The second class of materials attracts most research developments following the demonstration of spin-controlled OAM generation in the visible regime by geometric phase metasurfaces ${ }^{15,16}$. Meanwhile, textbook wave-mixing processes from homogeneous crystals have been extended to fields carrying OAM since the $1990 \mathrm{~s}^{17-19}$ and later to two-dimensional ${ }^{20}$ and three-dimensional ${ }^{21,22}$ structured nonlinear photonic crystals for the production of harmonic OAM states by design. Structured isotropic materials provide a versatile approach, merging optical spin-orbit coupling with nonlinear optics ${ }^{23}$.

Here we revisit the longstanding spin-selective second-harmonic wave (SHW) generated by a circularly polarized fundamental wave (FW) along the optical axis of a crystal with three-fold rotational symmetry in the realm of spin-orbit nonlinear wave mixing. We show that multiple spin-orbit optical states can be generated without relying on either artificial structuring of the photonic properties or on the non-paraxial regime, defined as a situation in which the longitudinal component of the field is explicitly at play. This contrasts with all experimental studies carried out so far in the paraxial regime, from the pioneering work ${ }^{2}$ to a more recent one $e^{24}$, both of which deal with collimated beams and report on the observation of cross-polarized SHW without any orbital signature, as expected from a plane wave treatment ${ }^{2}$. How the spin-orbit content of a FW carrying non-zero OAM is transformed into the SHW, however, remains unexplored and is the starting point of our study.

The experimental configuration is illustrated in Fig. 1a, where we introduce the spin-orbit optical states $(\sigma, \ell)_{\omega}$ of the fundamental (labelled $\omega$ ) and the harmonic (labelled $2 \omega$ ) waves as optical states, where $\sigma= \pm 1$ refers to the photon helicity that is related to left/right-handed circularly polarized states defined by the unit vectors $\mathbf{c}_{\sigma}=(\mathbf{x}+i \sigma \mathbf{y}) / \sqrt{2}$ and the integer $\ell$ refers to the topological charge of the on-axis optical phase singularity associated with a complex field amplitude proportional to $\exp (i \ell \phi)$ (where $\phi$ is the polar angle in the $(x, y)$ transverse plane). The experiment is carried out by irradiating a 5 -mm-thick $c$-cut $\beta$ - $\mathrm{BaB}_{2} \mathrm{O}_{4}$ (BBO) crys$\mathrm{tal}^{25}$ with a femtosecond laser source at $1,070 \mathrm{~nm}$ propagating along the optical axis of the crystal, which defines the $z$ axis. The incident spin state is prepared by using standard polarization optics, whereas the OAM state $\ell$ is prepared by using a helical phase mask (see Methods). The FW intensity profile at the midplane of the $\mathrm{BBO}$ crystal is imaged by a spherical lens and recorded by a camera; the value of $\ell$ is ascertained by replacing the spherical lens by a cylindrical lens ${ }^{26}$, see Fig. $1 \mathrm{~b}$ for $(\sigma, \ell)_{\omega}$ with $\sigma=+1$ and $\ell=\{0,-1,-2\}$ (see also Supplementary Figs. 1-4). The polarization state analysis of the SHW is found to be orthogonal to that of the incident FW whatever $\ell$ is, whereas its OAM state is identified as $2 \ell$, see Fig. $2 \mathrm{c}$ (see also Supplementary Figs. 5-8). This experimentally demonstrates that the second-harmonic generation from a collimated beam propagating along the optical axis follows the process $(\sigma, \ell)_{\omega} \rightarrow(-\sigma, 2 \ell)_{2 \omega}$.

This is supported analytically by looking at the FW-induced nonlinear polarization $P_{i}^{(2 \omega)}=\varepsilon_{0} \sum_{j k} \chi_{i j k}^{(2)} E_{j}^{(\omega)} E_{k}^{(\omega)}$, where $\varepsilon_{0}$ is the vacuum permittivity, $\chi_{i j k}^{(2)}$ is the quadratic susceptibility tensor and the indices $(i, j, k)$ run over the Cartesian coordinates $(x, y, z)$. Recalling the $C_{3 \mathrm{v}}$ symmetry group of the crystal and assuming that the principal mirror-plane is perpendicular to the $x$ axis, we have $\chi_{y y y}^{(2)}=-\chi_{y x x}^{(2)}=-\chi_{x x y}^{(2)}=-\chi_{x y x}^{(2)}=\chi^{(2)}$. Then, neglecting the longitudinal field according to our paraxial framework, we have $\mathbf{P}^{(2 \omega)}=\varepsilon_{0} \chi^{(2)} \frac{i \sigma}{\sqrt{2}}\left[\left(E_{x}^{(\omega)}+i \sigma E_{y}^{(\omega)}\right)^{2} \mathbf{c}_{\sigma}-\left(E_{x}^{(\omega)}-i \sigma E_{y}^{(\omega)}\right)^{2} \mathbf{c}_{-\sigma}\right]$. It is known that the helical phase profile imparted to the collimated Gaussian beam generates a hypergeometric-Gaussian mode that is expressed, up to a propagation phase factor, as $\mathbf{E}^{(\omega)} \propto u_{\ell}(r, z) \exp (i \ell \phi) \mathbf{c}_{\sigma}$, where $u_{\ell}(r, z)$ refers to the $z$-dependent slowly varying amplitude profile $^{26}$ whose radial dependence explains the observed rings in

'Department of Materials Science and Engineering, Southern University of Science and Technology, Shenzhen, China. ${ }^{2}$ Shenzhen Institute for Quantum Science and Engineering, Southern University of Science and Technology, Shenzhen, China. ${ }^{3}$ Université de Bordeaux, CNRS, LOMA, UMR 5798, Talence,

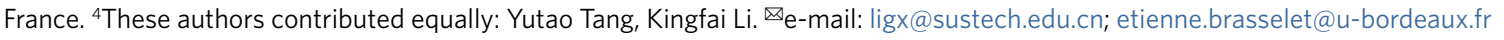




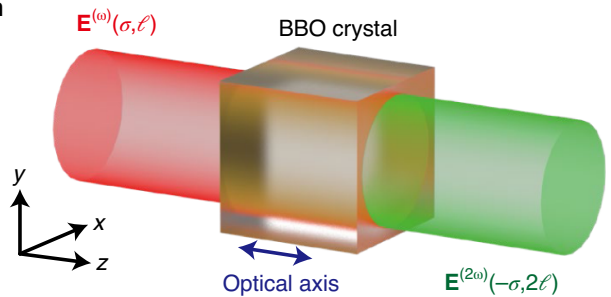

b
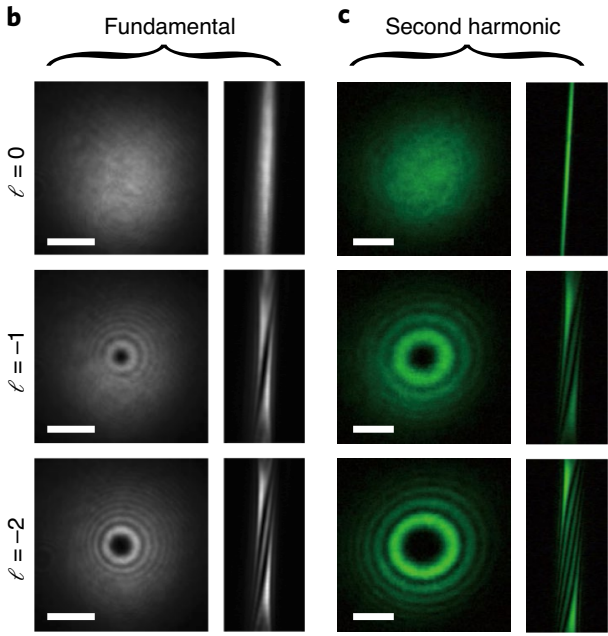

Fig. 1 | Spin-selective second-harmonic generation along the optical axis of a BBO crystal. a, Illustration of the light-matter interaction geometry when the nonlinear crystal is pumped by a collimated fundamental wave (red) with angular momentum state of $(\sigma, \ell)_{\omega}$ that generates a second harmonic wave (green) with angular momentum state of $(-\sigma, 2 \ell)_{2 \omega}$. The electric fields of the fundamental and second harmonic waves are labelled as $\mathbf{E}^{(\omega)}$ and $\mathbf{E}^{(2 \omega)}$, respectively. $\mathbf{b}$, Intensity profiles of the fundamental wave at the midplane of the crystal imaged using a spherical (left) or cylindrical (right) lens for $(\sigma, \ell)_{\omega}=(+1,0),(+1,-1)$ and $(+1,-2)$ (top to bottom). $\mathbf{c}$, Intensity profiles of the generated second-harmonic waves with angular momentum states of $(-1,0)_{2 \omega \prime}(-1,-2)_{2 \omega^{\prime}}$ and $(-1,-4)_{2 \omega}$ (top to bottom) imaged at the midplane of the crystal using a spherical (left) or cylindrical (right) lens. Scale bar, $1 \mathrm{~mm}$. the intensity profiles. This gives $\mathbf{P}^{(2 \omega)} \propto \chi^{(2)} \sigma u_{\ell}^{2}(r, z) \exp (2 i \ell \phi) \mathbf{c}_{-\sigma}$, which implies that a SHW with angular momentum state $(-\sigma, 2 \ell)_{2 \omega}$ is radiated, as observed experimentally.

The spin-orbit content of the SHW is markedly enriched as soon as the linear spin-orbit coupling process inherent to the propagation of focused light along the optical axis takes place-see Fig. 2a, which illustrates our second set of experiments. The birefringence of the nonlinear crystal is 'read' by a non-collimated paraxial beam even though it propagates along the optical axis, as its constituting plane waves travelling at oblique incidence angles experience the optical anisotropy. The axisymmetric nature of the light-matter interaction thus makes the crystal act as a space-variant uniaxial retarder with a topological charge of +1 regarding the azimuthal degree of free$\mathrm{dom}^{27}$. As expected from previous studies ${ }^{12,28}$, this is associated with an OAM increment by $2 \sigma$ for spin-flipped photons as the polarization state evolves in the course of propagation. The latter spin-orbit changes are substantial when the crystal length $L$ is larger than the anisotropic diffraction characteristic length $L_{\mathrm{c}}=\pi w_{0}^{2} n^{2} /(\lambda \mathrm{d} n)^{28}$, where $w_{0}$ is the beam waist, $\lambda$ is the wavelength, $n$ is the average refractive index of the crystal and $\mathrm{d} n$ its birefringence. With $L_{\mathrm{c}} \approx 0.6 \mathrm{~mm}$ for the fundamental wave, we choose an external $\mathrm{FW}$ Gaussian divergence angle of $0.113 \mathrm{rad}$, which converts approximately $50 \%$ of the incident wave power into the cross-polarization state (see Supplementary Fig. 9). Such a weakly focused beam is sufficient to efficiently combine paraxial second-harmonic generation with spin-orbit optical effects, as shown in Fig. $2 \mathrm{~b}$ for $\ell=\{0,-1\}$, where the identification of the spin-orbit states in the midplane of the sample (that is, where the FW is focused) is reported; see also Supplementary Figs. 10-12. In turn, each of the two FW states $(\sigma, \ell)_{0}$ and $(-\sigma, \ell+2 \sigma)_{\omega}$ can thus act as the sources of distinct harmonic states $(-\sigma, 2 \ell)_{2 \omega}$ and $(\sigma, 2 \ell+4 \sigma)_{2 \omega}$, respectively. The ensuing spinorbit interaction of latter states as they propagate through the second half of the crystal is thus expected to lead to another pair of harmonic states: $(\sigma, 2 \ell-2 \sigma)_{2 \omega}$ and $(-\sigma, 2 \ell+6 \sigma)_{2 \omega}$, respectively.

The overall process leading to four spin-orbit harmonic states from a single fundamental state is summarized in Fig. 3 (see the Supplementary Information for a simplified model). Experimentally, spin-resolved imaging of the SHW intensity profile hints at the latter harmonic spin-orbit cascade, as shown in Fig. 2c for $\ell=\{0,-1\}$ (see also Supplementary Figs. 13-16). Still,

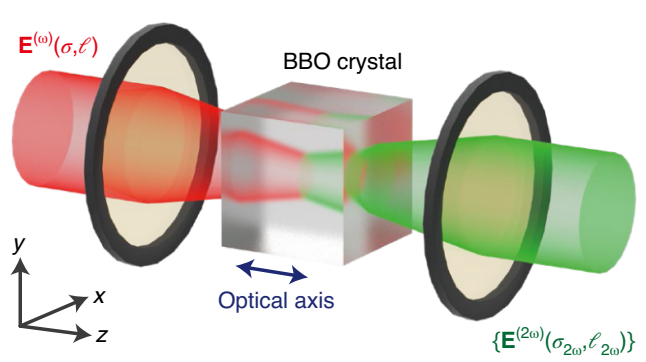

b

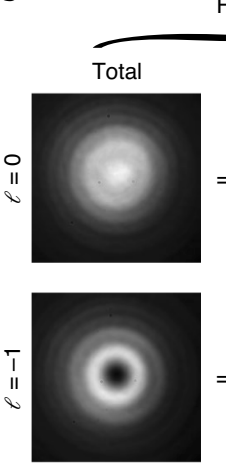

Fundamental $(\sigma=+1)$
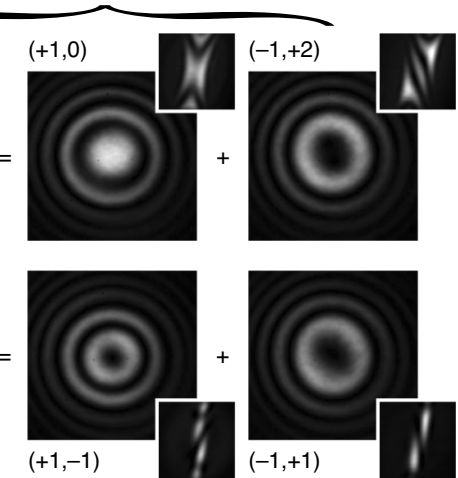

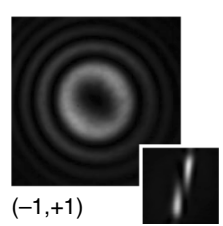

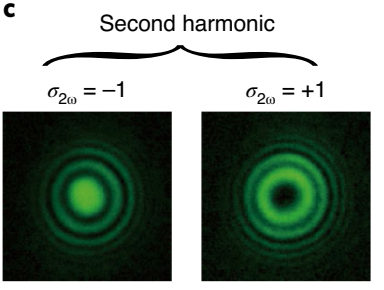
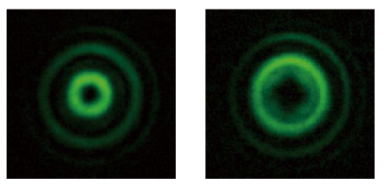

Fig. 2 | Spin-orbit angular momentum cascade along the optical axis of a BBO crystal with weakly focused pumping light. a, Illustration of the light-matter interaction geometry. The incident fundamental wave with angular momentum state of $(\sigma, \ell)$ generates a second-harmonic field comprising a set of distinct angular momentum states $\left(\sigma_{2 \omega} \ell_{2 \omega}\right)$. b. Left, total intensity profiles of the fundamental wave at the midplane of the crystal for incident optical states $(\sigma, \ell)_{\omega}=(+1,0)$ (top row) and $(\sigma, \ell)_{\omega}=(+1,-1)$ (bottom row) and external Gaussian divergence of 0.113 rad. Middle and right, corresponding intensity profiles of the two circularly polarized components imaged using either a spherical lens (main images) or a cylindrical lens (insets: the number and orientation of the dark fringes allow the magnitude and sign of the OAM state $\ell$ to be determined, respectively). c, Intensity profiles of the corresponding circularly polarized components of the generated second-harmonic field imaged using a spherical lens. Note that we do not discuss the OAM state of the SHW because here we only deal with polarization projection as a first step towards unveiling the spin-orbit content of the SHW. 


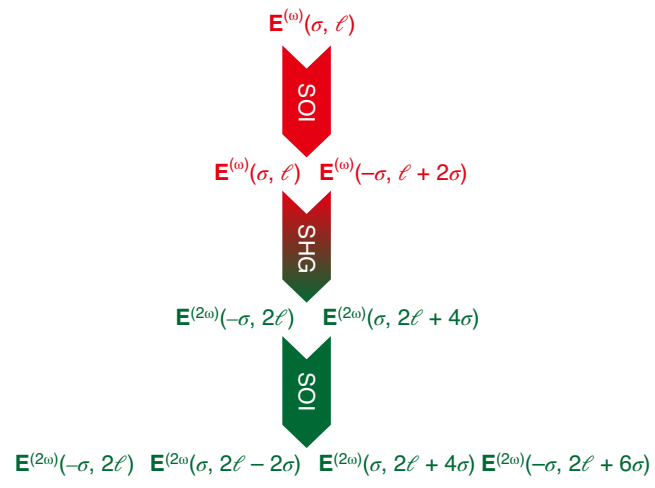

Fig. 3 | Three-step description of the spin-orbit angular momentum cascade for the second-harmonic generation process. As a paraxial incident fundamental wave (red) with angular momentum state $(\sigma, \ell)_{\omega}$ propagates, the generated second-harmonic field (green) constitutes up to four distinct angular momentum states $\left(\sigma_{2 \omega^{\prime}} \ell_{2 \omega}\right)$. The first step of the process is the generation of the state $(-\sigma, \ell+2 \sigma)_{\omega}$ via the linear spin-orbit interaction (SOI) experienced by the fundamental wave. The second step is the second-harmonic generation (SHG) itself, which relies on the nonlinear optics selection rules pertaining to circular polarization states and the conservation law for the orbital angular momentum per photon, and leads to states $(-\sigma, 2 \ell)_{2 \omega}$ and $(\sigma, 2 \ell+4 \sigma)_{2 \omega}$. The third step corresponds to linear SOI experienced by each of the circularly polarized components of the second-harmonic field, which produces the states $(\sigma, 2 \ell-2 \sigma)_{2 \omega}$ and $(-\sigma, 2 \ell+6 \sigma)_{2 \omega}$ quantitatively unveiling the spin-orbit content of the SHW requires an OAM-selective detection scheme. This is done by imparting an azimuthal phase factor $\exp (i \Delta \ell \phi)$ to each of the circularly polarized components of the SHW projection, which performs the operation $\left(\sigma_{2 \omega}, \ell_{2 \omega}\right) \rightarrow\left(\sigma_{2 \omega}, \ell_{2 \omega}+\Delta \ell\right)$. The identification of the OAM state $\ell_{2 \omega}$ is subsequently verified by the observation of on-axis spot in the focal plane of spherical lens ${ }^{29}$ for $\Delta \ell=-\ell_{2 \omega}$ (see Supplementary Fig. 17).

In Fig. 4a we show the results for an incident Gaussian FW with state $(+1,0)_{\omega}$, for which three of the four expected spinorbit states are detected (see red boxes): $(-\sigma, 2 \ell)_{2 \omega} \equiv(-1,0)_{2 \omega}$, $(\sigma, 2 \ell+4 \sigma)_{2 \omega} \equiv(+1,+4)_{2 \omega}$ and $(\sigma, 2 \ell-2 \sigma)_{2 \omega} \equiv(+1,-2)_{2 \omega}$. The state $(-\sigma, 2 \ell+6 \sigma)_{2 \omega} \equiv(-1,+6)_{2 \omega}$ is not accessible with our set-up, which is restricted to the range $-4 \leq \Delta \ell \leq+4$. Still, even this limited bandwidth for deciphering the OAM spectrum is enough to recover all of the predicted states in $\sigma \ell=-1$, the results of which are shown in Fig. $4 \mathrm{~b}$ for incident FW state $(+1,-1)_{\omega}$. Despite careful optimization of the optical alignment, the $(-\sigma, 2 \ell+6 \sigma)_{2 \omega} \equiv(-1,+4)_{2 \omega}$ is not found. Nevertheless, the observed broken axisymmetry that is all the more pronounced as $|\Delta \ell|$ increases (which is consistent with helical phase masks imperfections; see Supplementary Fig. 18) could explain the difficulty in experimentally unravelling the state $(-\sigma, 2 \ell+6 \sigma)_{2 \omega}$ that is at the 'end' of the harmonic spin-orbit angular momentum cascade (see Fig. 3). Similar results are found for the incident FW states $(-1,0)_{\omega}$ and $(-1,+1)_{\omega}$, see Supplementary Fig. 19, and simulations from our simplified model are summarized in Supplementary Figs. 20 and 21.

The interplay between the spin-orbit coupling and nonlinear wave mixing illustrated here in the framework of second-harmonic a
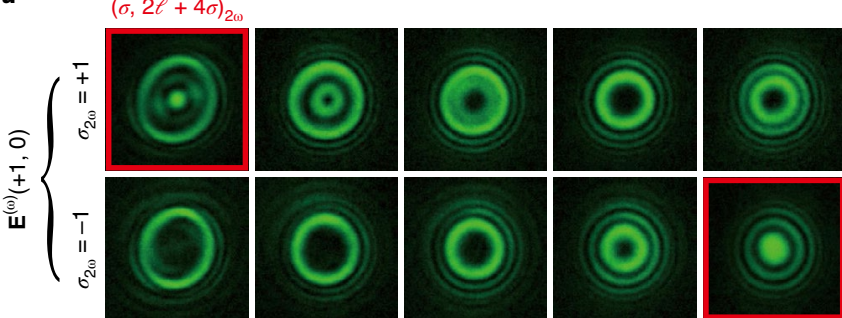

$(-\sigma, 2 \ell)_{2 \omega}$ $(\sigma, 2 \ell-2 \sigma)_{2 \omega}$
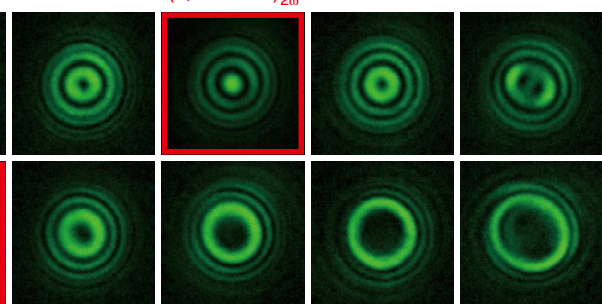

0

1

2

3

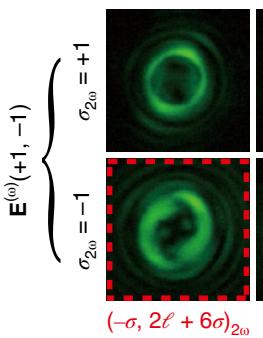

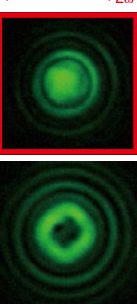

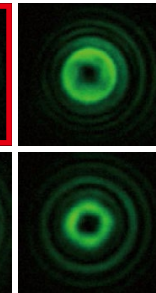

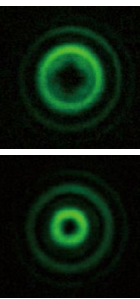

$\circ$

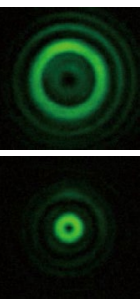

$\odot$

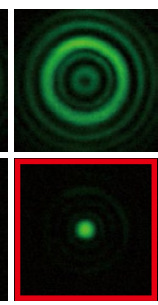

$(-\sigma, 2 \ell)_{2 \omega}$ $(\sigma, 2 \ell-2 \sigma)_{2 \omega}$

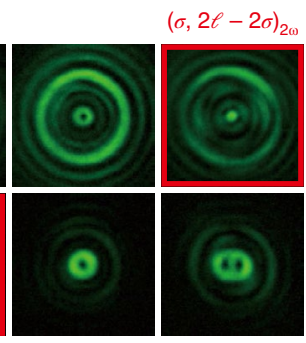

๑

Fig. 4 | Spin-orbit tomography of the second-harmonic field for weakly focused pumping light. The experimental set-up is presented in Fig. 2. The spin-orbit optical content of the second-harmonic field is retrieved by imparting a set of helical phase profiles with topological charge $\Delta \ell$ to each of the circularly polarized field components by using a set of azimuthally varying half-wave plates followed by optical Fourier transform performed by a spherical lens. $\mathbf{a}$, The incident fundamental optical state is $(\sigma, \ell)_{\omega}=(+1,0)$. Three out of the four angular momentum states $(-1,0)_{2 \omega^{\prime}}(+1,+4)_{2 \omega}$ and $(+1,-2)_{2 \omega}$ are identified as on-axis intensity spots (see red boxes), whereas the fourth falls outside the experimentally accessible OAM range. $\mathbf{b}$, The incident fundamental optical state is $(\sigma, \ell)_{\omega}=(+1,-1)$. Three out of the four angular momentum states $(-1,-2)_{2 \omega \prime}(+1,+2)_{2 \omega}$ and $(+1,-4)_{2 \omega}$ are identified (see red solid boxes), whereas the fourth predicted state is not observed (see the red dashed box). All of the intensity profiles are normalized to the maximal intensity values in each subplot. 
generation and crystals with $C_{3 \mathrm{v}}$ symmetry can be extended both to high-harmonic processes and other discrete rotational symmetries, for which circular polarization selection rules have been previously established for non-zero OAM and in the absence of spin-orbit interactions mediated by the optical anisotropy ${ }^{30}$. Namely, using an incident collimated beam with state $(\sigma, \ell)_{\omega}$ that propagates along the optical axis of a crystal with $m$-fold rotational symmetry, the order $n$ of the allowed circularly polarized harmonics satisfies $n=p m \pm 1$, where $p$ is a positive integer, and \pm refers to $\pm \sigma$ circularly polarized high-harmonic waves. When the incident $\mathrm{FW}$ is focused, a secondary FW state $(-\sigma, \ell+2 \sigma)_{\omega}$ arises from spin-orbit coupling and the following spin-orbit arithmetic is anticipated. The states of the high-harmonic waves of order $n=p m+1$ are $(\sigma, n \ell)_{n \omega}$ and $(-\sigma$, $n \ell+2 n \sigma)_{n \omega}$, which further lead to the spin-orbit cascaded states $(-\sigma, n \ell+2 \sigma)_{n \omega}$ and $(\sigma, n \ell+2(n-1) \sigma)_{n \omega}$, respectively. The states of the high-harmonic waves of order $n=p m-1$ are $(-\sigma, n \ell)_{n \omega}$ and $(\sigma, n \ell+2 n \sigma)_{n \omega}$, which further lead to the spin-orbit cascaded states $(\sigma, n \ell-2 \sigma)_{n \omega}$ and $(-\sigma, n \ell+2(n+1) \sigma)_{n \omega}$, respectively. The situation is summarized in Supplementary Tables 1-8 for crystals with various discrete rotational symmetries.

Interestingly, the non-paraxial regime-where the longitudinal field component enters explicitly in the second-harmonic generation process-has been explored in a couple of previous works ${ }^{31-33}$ with experimental demonstration of azimuthally modulated total SHW intensity. However, there are only theoretical predictions regarding the spin-orbit content of the harmonic field generated from non-paraxial Bessel beams: a FW characterized by a transverse state $(\sigma, 0)_{\omega}$ has been predicted to lead to SHW transverse states $(-\sigma, 0)_{2 \omega},(\sigma,-\sigma)_{2 \omega},(-\sigma, 3 \sigma)_{2 \omega}$ and $(\sigma, 4 \sigma)_{2 \omega}$, where the states with OAM components $\ell_{2 \omega}=-\sigma$ and $3 \sigma$ are of a non-paraxial nature ${ }^{31-33}$. In contrast, we demonstrate that the paraxial regime allows axisymmetric harmonic intensity distributions to be obtained. Moreover, the experimental spin-orbit tomography of the SHW reveals unpredicted angular momentum states.

Extending the present results to the non-paraxial regime is a task for a future work, with the aim of reaching phase-matching conditions $^{31-33}$ and thereby higher efficiencies in harmonic generation. Another option to increase efficiency could be to implement a single-pass quasi-phase-matching process $^{34}$ or an intracavity design $^{6}$. Either way, the rich physics observed in this work shows that the re-examination of nonlinear optical processes in crystals is worthwhile. In particular, noting that the long-established selection rules pertaining to circular polarization states of harmonic generation in bulk crystals ${ }^{30}$ also apply to $2 \mathrm{D}$ metacrystals ${ }^{35,36}$, a flat-optics version of the present work seems possible via multiplexing nonlinear and multiple-wavelength spin-orbit features into a single metadevice. These results also provide possible routes to controlling the multiplexed angular momentum states of light ${ }^{37}$, which may have important applications in areas such as high-dimensional quantum information processing ${ }^{38,39}$, optical pulses with frequency-dependent $\mathrm{OAM}^{40}$ and multi-state optical magnetization ${ }^{8}$.

\section{References}

1. Bliokh, K. Y., Rodríguez-Fortuño, F. J., Nori, F. \& Zayats, A. V. Spin-orbit interactions of light. Nat. Photon. 9, 796-808 (2015).
2. Simon, H. J. \& Bloembergen, N. Second-harmonic light generation in crystals with natural optical activity. Phys. Rev. 171, 1104-1114 (1968).

3. Patel, C. K. N. \& Van Tran, N. Phase-matched nonlinear interaction between circularly polarized waves. Appl. Phys. Lett. 15, 189-191 (1969).

4. Wang, J. et al. Terabit free-space data transmission employing orbital angular momentum multiplexing. Nat. Photon. 6, 488-496 (2012).

5. Omatsu, T., Miyamoto, K. \& Lee, A. J. Wavelength-versatile optical vortex lasers. J. Opt. 19, 123002 (2017).

6. Sroor, H. et al. High-purity orbital angular momentum states from a visible metasurface laser. Nat. Photon. 14, 498-503 (2020).

7. Fleischer, A., Kfir, O., Diskin, T., Sidorenko, P. \& Cohen, O. Spin angular momentum and tunable polarization in high-harmonic generation. Nat. Photon. 8, 543-549 (2014).

8. Lin, S. et al. All-optical vectorial control of multistate magnetization through anisotropy-mediated spin-orbit coupling. Nanophotonics 8, 2177-2188 (2019).

9. Allen, L., Beijersbergen, M. W., Spreeuw, R. J. C. \& Woerdman, J. P. Orbital angular momentum of light and the transformation of Laguerre-Gaussian laser modes. Phys. Rev. A 45, 8185-8189 (1992).

10. Shen, Y. et al. Optical vortices 30 years on: OAM manipulation from topological charge to multiple singularities. Light Sci. Appl. 8, 90 (2019).

11. Volyar, A. V., Fadeeva, T. A. \& Egorov, Yu. A. Vector singularities of Gaussian beams in uniaxial crystals: optical vortex generation. Tech. Phys. Lett. 28, 70-77 (2002).

12. Ciattoni, A., Cincotti, G. \& Palma, C. Circularly polarized beams and vortex generation in uniaxial media. J. Opt. Soc. Am. A 20, 163-171 (2003).

13. Biener, G., Niv, A., Kleiner, V. \& Hasman, E. Formation of helical beams by use of Pancharatnam-Berry phase optical elements. Opt. Lett. 27, 1875-1877 (2002).

14. Marrucci, L., Manzo, C. \& Paparo, D. Optical spin-to-orbital angular momentum conversion in inhomogeneous anisotropic media. Phys. Rev. Lett. 96, 163905 (2006).

15. Li, G. et al. Spin-enabled plasmonic metasurfaces for manipulating orbital angular momentum of light. Nano Lett. 13, 4148-4151 (2013).

16. Karimi, E. et al. Generating optical orbital angular momentum at visible wavelengths using a plasmonic metasurface. Light Sci. Appl. 3, e167 (2014).

17. Basistiy, I. V., Bazhenov, V. Y., Soskin, M. S. \& Vasnetsov, M. V. Optics of light beams with screw dislocations. Opt. Commun. 103, 422-428 (1993).

18. Dholakia, K., Simpson, N. B., Padgett, M. J. \& Allen, L. Second-harmonic generation and the orbital angular momentum of light. Phys. Rev. A 54, R3742-R3745 (1996).

19. Beržanskis, A., Matijošius, A., Piskarskas, A., Smilgevičius, V. \& Stabinis, A. Sum-frequency mixing of optical vortices in nonlinear crystals. Opt. Commun. 150, 372-380 (1998).

20. Bloch, N. V. et al. Twisting light by nonlinear photonic crystals. Phys. Rev. Lett. 108, 233902 (2012).

21. Liu, S. et al. Nonlinear wavefront shaping with optically induced three-dimensional nonlinear photonic crystals. Nat. Commun. 10, 3208 (2019).

22. Wei, D. et al. Efficient nonlinear beam shaping in three-dimensional lithium niobate nonlinear photonic crystals. Nat. Commun. 10, 4193 (2019).

23. Li, G., Zhang, S. \& Zentgraf, T. Nonlinear photonic metasurfaces. Nat. Rev. Mater. 2, 17010 (2017)

24. Li, G., Zentgraf, T. \& Zhang, S. Rotational Doppler effect in nonlinear optics. Nat. Phys. 12, 736-740 (2016).

25. Chen, C., Wu, B., Jiang, A. \& You, G. A new-type ultraviolet SHG crystal: $\beta$ - $\mathrm{BaB}_{2} \mathrm{O}_{4}$. Sci. Sin. Ser. B 28, 235-243 (1985).

26. Bekshaev, A. Y., Soskin, M. S. \& Vasnetsov, M. V. Transformation of higher-order optical vortices upon focusing by an astigmatic lens. Opt. Commun. 241, 237-247 (2004).

27. Loussert, C. \& Brasselet, E. Efficient scalar and vectorial singular beam shaping using homogeneous anisotropic media. Opt. Lett. 35, 7-9 (2010)

28. Brasselet, E. et al. Dynamics of optical spin-orbit coupling in uniaxial crystals. Opt. Lett. 34, 1021-1023 (2009).

29. Langford, N. K. et al. Measuring entangled qutrits and their use for quantum bit commitment. Phys. Rev. Lett. 93, 053601 (2004).

30. Bhagavantam, S. \& Chandrasekhar, P. Harmonic generation and selection rules in nonlinear optics. Proc. Ind. Acad. Sci. A 76, 13-20 (1972).

31. Belyi, V., Khilo, N., Forbes, A. \& Ryzhevich, A. Generation and propagation of high-order Bessel vortices in linear and non-linear crystals. In Proc. SPIE 7430, Laser Beam Shaping X 74300F (SPIE, 2009).

32. Sato, S. \& Kozawa, Y. Radially polarized annular beam generated through a second-harmonic-generation process. Opt. Lett. 34, 3166-3168 (2009).

33. Belyi, V., Khilo, N., Kazak, N., Ryzhevich, A. \& Forbes, A. Propagation of high-order circularly polarized Bessel beams and vortex generation in uniaxial. Opt. Eng. 50, 059001 (2011).

34. Shao, G.-H., Wu, Z.-J., Chen, J.-H., Xu, F. \& Lu, Y.-Q. Nonlinear frequency conversion of fields with orbital angular momentum using quasi-phase-matching. Phys. Rev. A 88, 063827 (2013). 
35. Chen, S. et al. Symmetry-selective third-harmonic generation from plasmonic metacrystals. Phys. Rev. Lett. 113, 033901 (2014).

36. Konishi, K. et al. Polarization-controlled circular second-harmonic generation from metal hole arrays with threefold rotational symmetry. Phys. Rev. Lett. 112, 135502 (2014).

37. Buono, W. T. et al. Polarization-controlled orbital angular momentum switching in nonlinear wave mixing. Opt. Lett. 43, 1439-1442 (2018).

38. Wang, K. et al. Quantum metasurface for multiphoton interference and state reconstruction. Science 361, 1104-1108 (2018).
39. Stav, T. et al. Quantum entanglement of the spin and orbital angular momentum of photons using metamaterials. Science 361, 1101-1104 (2018).

40. Rego, L. et al. Generation of extreme-ultraviolet beams with time-varying orbital angular momentum. Science 364, eaaw9486 (2019). 


\section{Methods}

Nonlinear crystal. Both the linear spin-orbit interaction of the fundamental wave and the second-harmonic-generation process are experimentally studied in the cases of a collimated beam or weakly focused (paraxial) fundamental waves propagating through along the $c$ axis of a 5-mm-long $\beta$-BBO crystal whose extraordinary (e) and ordinary (o) refractive indices at FW and SHW wavelengths are $n_{e}^{(\omega)}=1.543, n_{o}^{(\omega)}=1.655$ and $n_{e}^{(2 \omega)}=1.556$, respectively (http://www.castech $\mathrm{com} /$ product/120.html).

Fundamental light source. A femtosecond laser (pulse duration: $\sim 250 \mathrm{fs}$, repetition rate: $80 \mathrm{MHz}$, central wavelength: $820 \mathrm{~nm}$ ) is used to pump an optical parametric oscillator (Coherent Chameleon OPO) and the output laser has a wavelength of $1,070 \mathrm{~nm}$. The spot size of the collimated laser beam is $\sim 4.3 \mathrm{~mm}$ in diameter. The left-handed $(\sigma=+1)$ or right-handed $(\sigma=-1)$ circular polarization state of the fundamental wave is prepared by using a linear polarizer and a quarter-wave plate. Non-zero orbital states are prepared by using azimuthally varying liquid crystal polymer half-wave plates designed for a wavelength of $1,064 \mathrm{~nm}$ (Thorlabs). In the weakly focused situation, the collimated laser beam is focused in the midplane of the nonlinear crystal by an underfilled objective lens with numerical aperture $\mathrm{NA}=0.25$. The output (fundamental and harmonic) light is recollimated by a second objective lens that is identical to the first one (see Fig. 2a).

Second-harmonic experiments. The spin-orbit states of the fundamental wave at the output of the crystal are characterized by standard polarization projection (spin state detection) and the cylindrical lens method ${ }^{28}$ (orbital state detection). The spin-orbit states of the harmonic wave are ascertained by combining circular polarization projection with a spatial mode analysis using the combination of a helical phase mask with optical Fourier transformation using a spherical lens (with a focal length of $100 \mathrm{~mm}$ ) with a camera placed at the focus ${ }^{29}$. In the latter case, the phase masks are azimuthally varying nanostructured glass half-wave plates designed for a wavelength of $532 \mathrm{~nm}$ (Altechna). In all cases, the second-harmonic-generation process deals with collinear fundamental and harmonic fields and does not satisfy phase-matching conditions since all of the paraxial beams propagate along the optical axis of the crystal. The efficiencies are measured as the ratio between the generated harmonic wave power and the incident fundamental wave power. In the case of the collimated FW, we measured a second-harmonic-generation efficiency of $2.7 \times 10^{-11}$ with an input power of $41 \mathrm{~mW}$, whereas in the focused FW case the second-harmonic-generation efficiency increases up to $8.2 \times 10^{-8}$ with a pumping power of $2.8 \mathrm{~mW}$.

\section{Data availability}

The data that support the plots within this paper and other findings of this study are available from the corresponding authors on reasonable request.

\section{Acknowledgements}

G.L. is financially supported by the National Natural Science Foundation of China (grant numbers 91950114 and 11774145), a Guangdong Provincial Innovation and Entrepreneurship Project (2017ZT07C071) and the Qiu Shi Science \& Technologies Foundation.

\section{Author contributions}

E.B. and G.L. proposed the idea and designed the experiment. Y.T., K.L., J.D., X.Z. and G.L. conducted the nonlinear optical measurements. E.B., G.L., Y.T. and J.D. wrote the manuscript. All authors participated in the data analysis and discussions. G.L. and E.B. supervised the project.

\section{Competing interests}

The authors declare no competing interests.

\section{Additional information}

Supplementary information Supplementary information is available for this paper at https://doi.org/10.1038/s41566-020-0691-0.

Correspondence and requests for materials should be addressed to G.L. or E.B. 


\title{
Supplementary Information for
}

\section{Harmonic spin-orbit angular momentum cascade in nonlinear optical crystals}

\author{
Yutao Tang ${ }^{1, \#}$, Kingfai $\mathrm{Li}^{1, \#}$, Xuecai Zhang ${ }^{1}$, Junhong Deng ${ }^{1,2}$, Guixin $\mathrm{Li}^{1,2 *}$, Etienne \\ Brasselet $^{3 *}$
}

${ }^{1}$ Department of Materials Science and Engineering, Southern University of Science and Technology, Shenzhen, 518055, China.

${ }^{2}$ Shenzhen Institute for Quantum Science and Engineering, Southern University of Science and Technology, Shenzhen, 518055, China.

${ }^{3}$ Université de Bordeaux, CNRS, LOMA, UMR 5798, F-33400 Talence, France.

${ }^{\#}$ These authors contributed equally to this work.

*e-mail: ligx@sustech.edu.cn; etienne.brasselet@u-bordeaux.fr

\section{Contents}

S1. Optical Spin and Orbital Changes for the Collimated FW .............................................2

S2. Second Harmonic Generations for the Collimated FW .....................................................5

S3. Spin-Orbit Coupling Experienced by a Focused FW in the Paraxial Regime.................... 7

S4. Spin-Resolved SHG for Focused FW in the Paraxial Regime ........................................ 10

S5. Spin-Orbit State Tomography of SHW for a Focused FW in the Paraxial Regime .......... 12

S6. Simplified Model and Spin-Orbit Tomography Simulations.......................................... 14

S7. Angular Momentum States of $n^{\text {th }}$ Harmonic Generation Waves....................................... 18 


\section{S1. Optical Spin and Orbital Changes for the Collimated FW}

Firstly, we examine the linear optical spin-orbit interaction process in a c-cut BBO ("c-cut" label being omitted in what follows) crystal for the case of a collimated FW. The measurement setup is shown in Fig. $\mathrm{S} 1$, the angular momentum states $\left(\sigma_{\omega}= \pm 1, \ell_{\omega}=\{0, \pm 1, \pm 2\}\right)$ of the FW are controlled by using the circular polarizer and azimuthally-varying half-wave plate (so called $q$-plate, see Methods for the details of the used $q$-plates). The results are shown in Figs. $\mathrm{S} 2-\mathrm{S} 4$, which reveals that the spin-orbit coupling is rather weak in the BBO crystal when the incident $\mathrm{FW}$ is collimated when propagating along the optical axis of the nonlinear crystal.

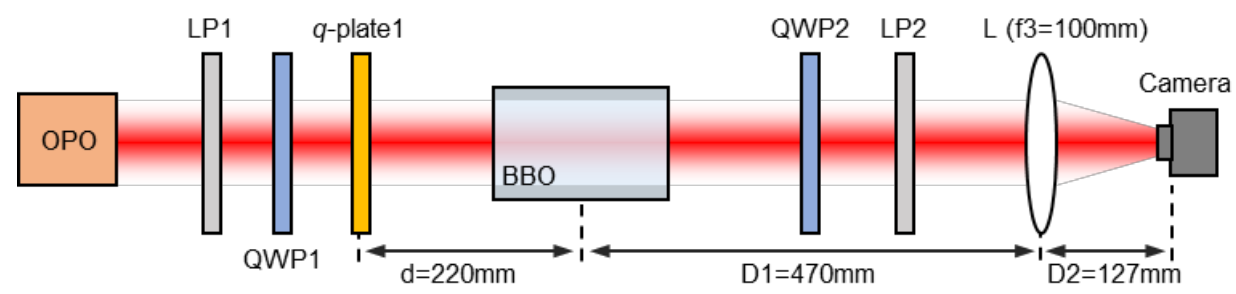

Fig. S1| Optical setup for assessing the spin and orbital changes in a BBO crystal (collimated case). OPO, optical parametric oscillator; LP, linear polarizer; QWP, quarter-wave plate; L, spherical lens. LP1 \& QWP1 together form a circular polarizer, while QWP2 \& LP2 form a circular analyzer. The $q$-platel is used to control the OAM states of the FW. The spherical lens L is dedicatedly placed to image the intensity profiles of the FW at the mid-plane of the BBO crystal.
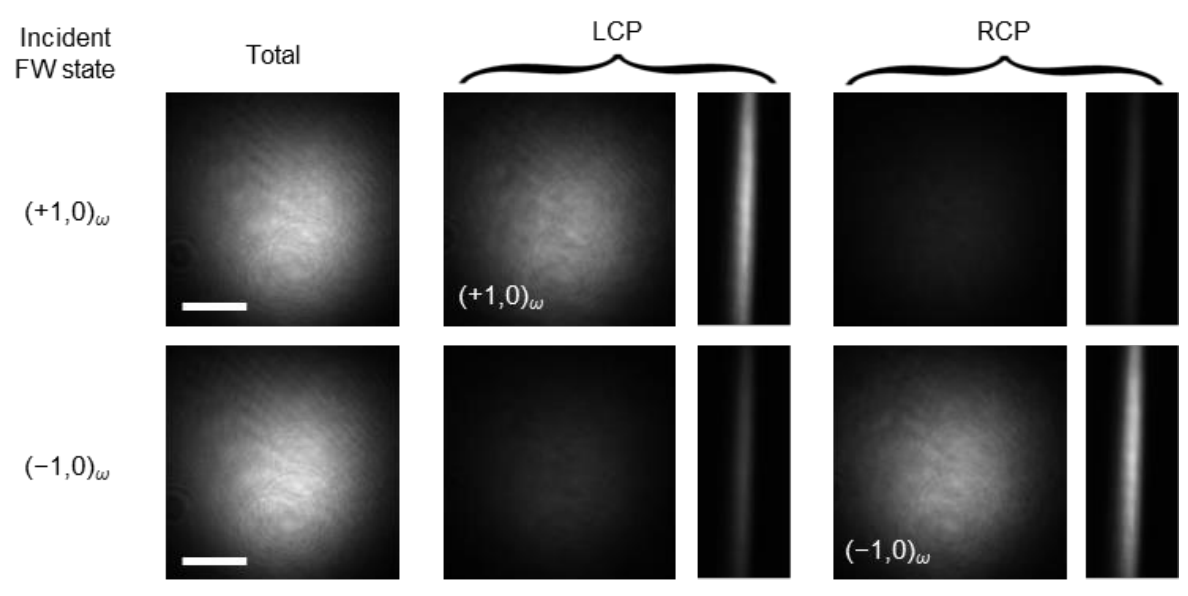

Fig. S2| Assessing the spin and orbital changes in the BBO crystal under the illumination of a collimated Gaussian FW $\left(\boldsymbol{\ell}_{\boldsymbol{\omega}}=\mathbf{0}\right)$. The incident FW state is denoted as $(\sigma, \ell)_{\omega}$, where $\sigma$ represents the spin state and $\ell$ represents the orbital state. The total intensity profiles as well as the left and right circularly polarized (LCP/RCP) components are measured (square images). The intensity profiles of LCP or RCP components imaged by a cylindrical lens are used to identify the OAM values (rectangle images). Scale bars: $1 \mathrm{~mm}$. 

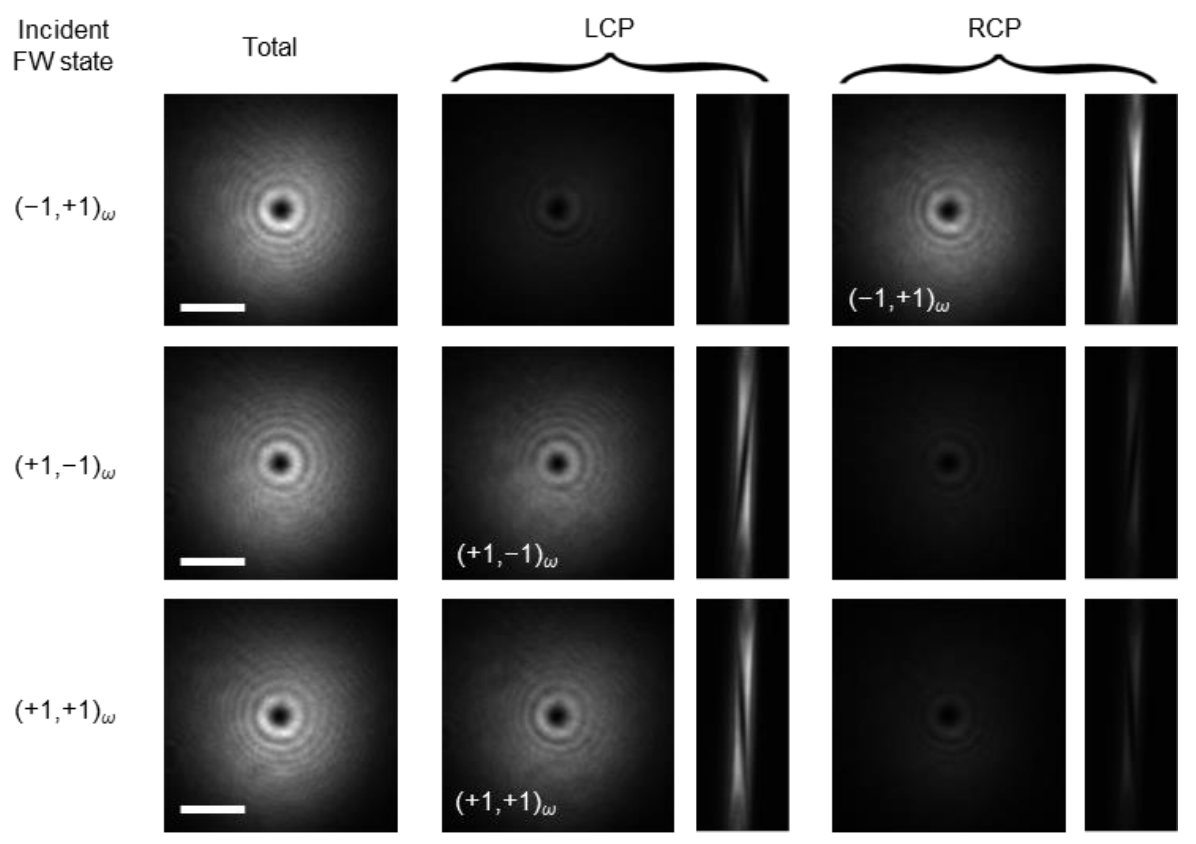

$(-1,-1)_{\omega}$
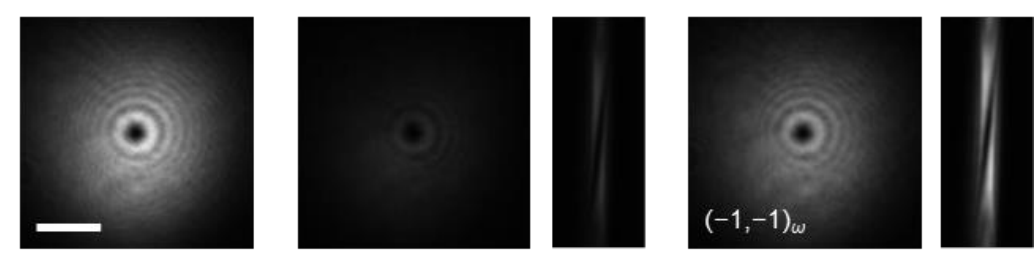

Fig. S3| Assessing the spin and orbital changes in the BBO crystal under the illumination of a collimated FW with angular momentum state of $( \pm \mathbf{1}, \pm \mathbf{1})_{\boldsymbol{\omega}}$. Scale bars: $1 \mathrm{~mm}$. Similarly, the OAM states can be obtained by using a cylindrical lens mode converter. Scale bars: $1 \mathrm{~mm}$. 


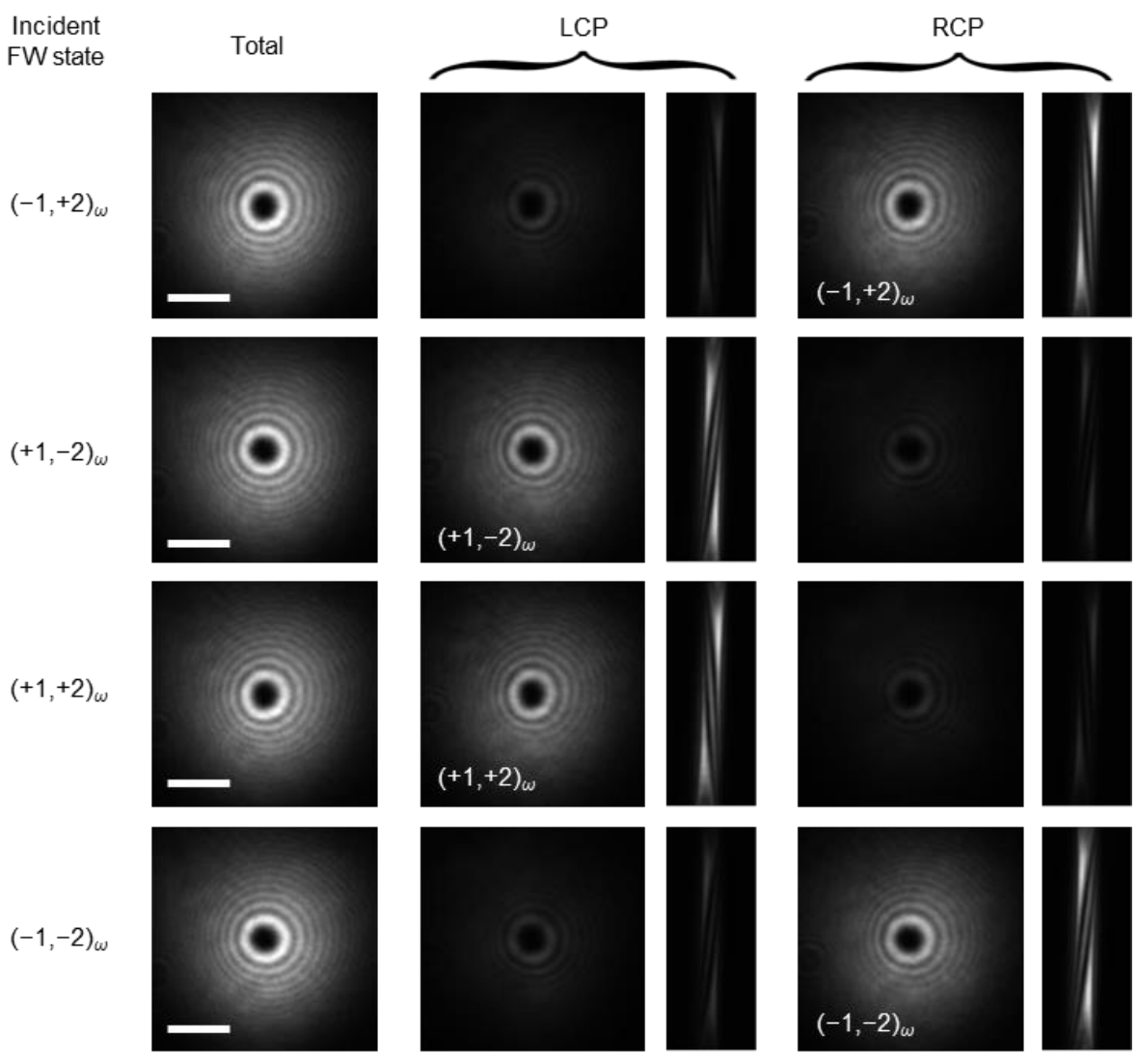

Fig. S4| Assessing the spin and orbital changes in the BBO crystal under the illumination of a collimated FW with angular momentum state $( \pm \mathbf{1}, \pm \mathbf{2})_{\omega}$. Scale bars: $1 \mathrm{~mm}$. 


\section{S2. Second Harmonic Generations for the Collimated FW}

When the FW propagates along the optical axis of a BBO crystal, the second harmonic generation ( $\mathrm{SHG}$ ) can be induced due to the inversion symmetry breaking of the crystal. In Fig. S5, a typical spectrum of the SHG signal wave is presented, as well as the power dependent SHG intensity, from which the second-order nonlinear SHG process can be experimentally confirmed.

The conservation of OAM during the SHG process is experimentally demonstrated by using a collimated FW pumping along the c-axis (optical axis) of the crystal (Figs. S6-S8). The experimental setup is shown in Fig. S6, and the results are demonstrated in Figs. S7 and S8, where the co-polarization components of SHG are forbidden due to the symmetry selection rules in nonlinear optics. The OAM values are ascertained by the dark fringes of the intensity profiles, which are imaged using a cylindrical lens. Through the comparison between Figs. S7 $\&$ S8 and Figs. S3 \& S4, one can simply conclude the nonlinear process $(\sigma, \ell)_{\omega} \rightarrow(-\sigma, 2 \ell)_{2 \omega}$.
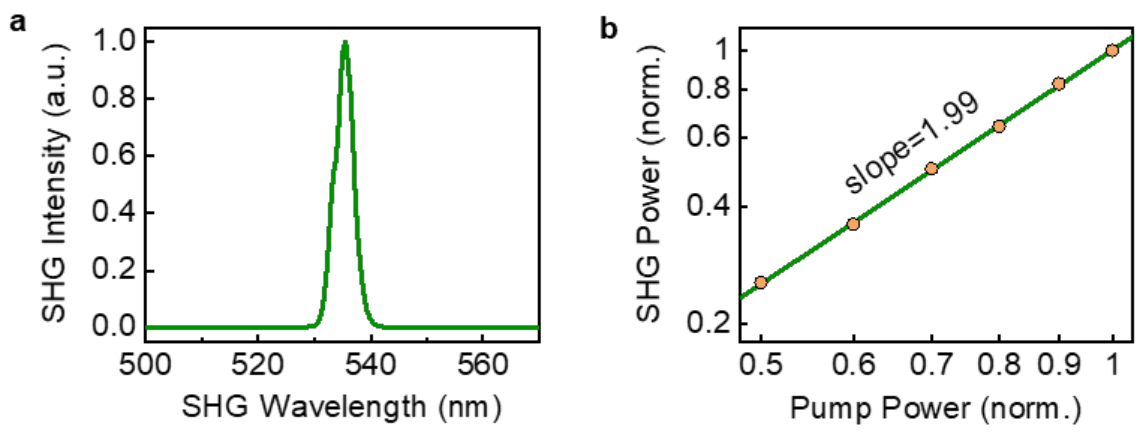

Fig. S5 $\mid$ a, A typical spectrum of the SHG signal from the BBO crystal as recorded by a fiber coupled spectrometer (Ocean Optics). The central wavelength of the spectrum is $\sim 535 \mathrm{~nm}$, corresponding to the fundamental wavelength of $1070 \mathrm{~nm}$. b. Power dependent SHG intensities from the BBO crystal. The axes are in log scales. The linear fitting of the experimental data gives a slope of 1.99 , indicating a second-order nonlinear optical process.

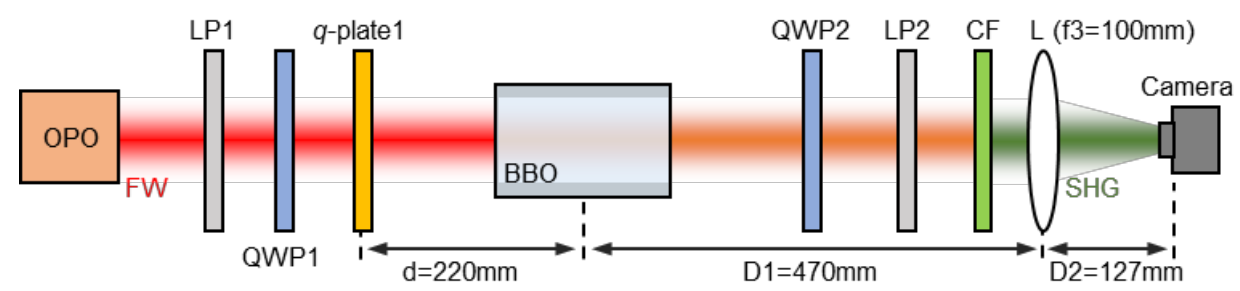

Fig. S6| Optical setup for the observation of second harmonic generations in a BBO crystal (collimated FW). OPO, optical parametric oscillator; LP, linear polarizer; QWP, quarter-wave plate; $\mathrm{CF}$, color filter to filter out the $\mathrm{FW}$ at $1070 \mathrm{~nm}$; L, spherical lens. LP1 \& QWP1 together form a circular polarizer, while QWP2 \& LP2 form a circular analyzer. The $q$ plate1 is used to generate the OAM states of the FW. The Lens L is dedicatedly placed to image the intensity profiles of the SHW at the mid-plane of the BBO crystal. 


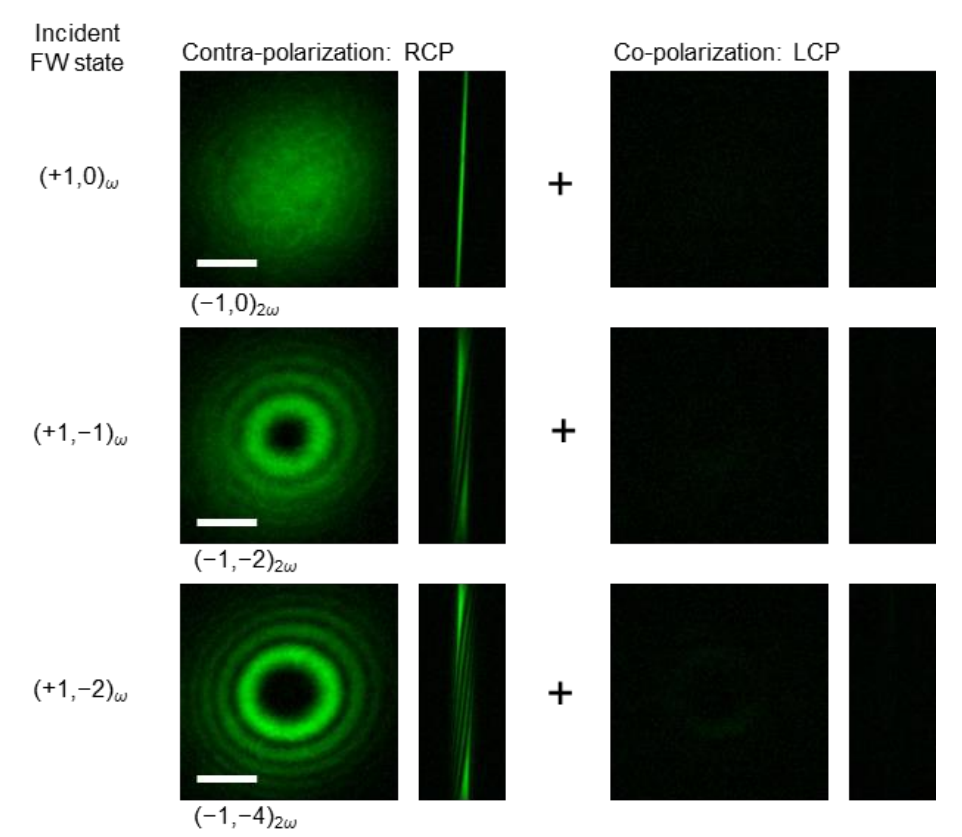

Fig. S7| SHWs from the BBO crystal excited by the collimated OAM-carrying or Gaussian FWs. The FWs are left circularly polarized $\left(\sigma_{\omega}=+1\right)$. The profiles of SHWs with contra- and co-polarization states to that of the FWs are imaged using either a spherical or a cylindrical lens. $(\sigma, \ell)_{\omega}$ and $(\sigma, \ell)_{2 \omega}$ denote the spin and orbital angular momentum states of the incident FWs and the output SHWs, respectively. Scale bars: $1 \mathrm{~mm}$.

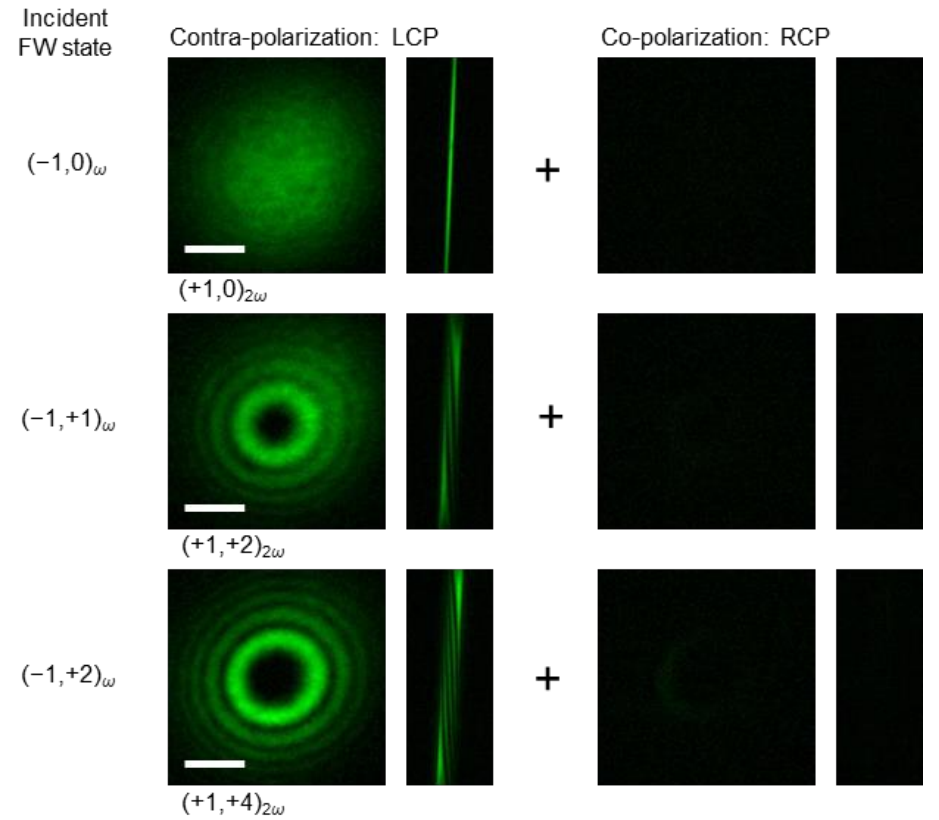

Fig. S8 $\mid$ SHWs from the BBO crystal excited by the collimated OAM-carrying or Gaussian FWs. The FWs are left circularly polarized $\left(\sigma_{\omega}=-1\right)$. The profiles of SHWs with contra- and co-polarization states to that of the FWs are imaged using either a spherical or a cylindrical lens. $(\sigma, \ell)_{\omega}$ and $(\sigma, \ell)_{2 \omega}$ denote the spin and orbital angular momentum states of the incident FWs and the output SHWs, respectively. Scale bars: $1 \mathrm{~mm}$. 


\section{S3. Spin-Orbit Coupling Experienced by a Focused FW in the Paraxial}

\section{$\underline{\text { Regime }}$}

In this section, we are interested in the spin-orbital angular momentum conversion of the focused FW in the paraxial regime. Fig. S9 shows the beam divergences of the FW and SHW as well as the spin-to-orbital conversion efficiencies against various external divergence angles of the FWs. Note that our experiments in are conducted using $\theta_{\mathrm{FW}}=0.113$, which is certainly paraxial, and for which we measured a conversion efficiency of 0.48 for the FW field. In Fig. S10, we show the experiment setup to characterize the angular momentum states of the output FWs from the BBO crystal. The results are shown in Figs. S11 $\left(\ell_{\omega}=0\right)$ and S12 $\left(\ell_{\omega}= \pm 1\right)$. Two FW states, as results of the spin-orbit coupling effects, can be identified as the incident one ( $\sigma$, $\ell)_{\omega}$ and the SOI-induced one $(-\sigma, \ell+2 \sigma)_{\omega}$.

a

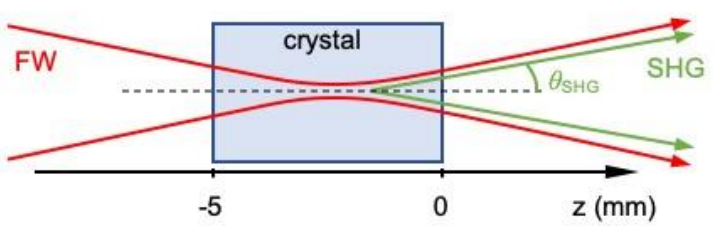

b

\begin{tabular}{ccc}
\hline $\mathrm{f}$ & $\theta_{\mathrm{FW}}(\mathrm{rad})$ & $\theta_{\mathrm{SHG}}(\mathrm{rad})$ \\
\hline $10 \mathrm{~mm}$ & 0.113 & 0.0415 \\
$35 \mathrm{~mm}$ & 0.052 & 0.0325 \\
$50 \mathrm{~mm}$ & 0.038 & 0.0275 \\
\hline
\end{tabular}

C

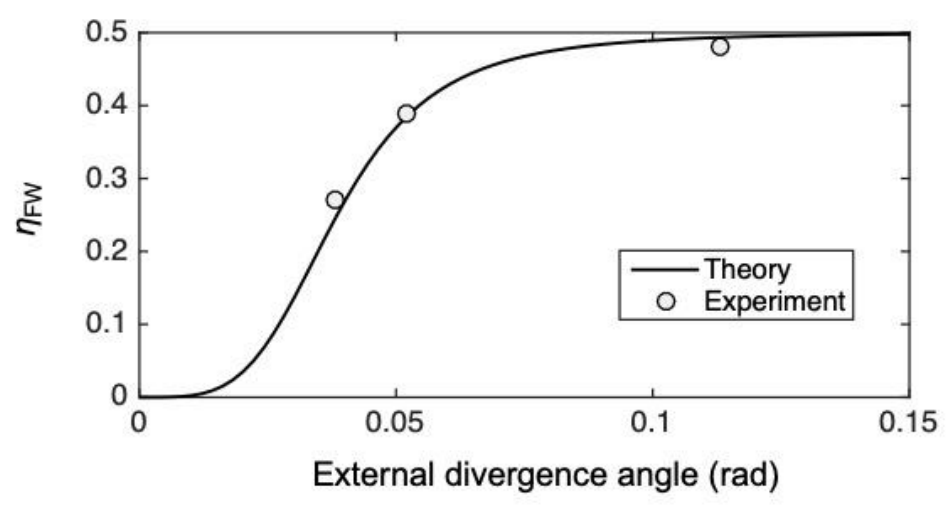

Fig. S9| Beam divergences and the linear spin-to-orbital angular momentum conversion. The incident FW is left circularly polarized Gaussian beam. a, Scheme of the experimental configuration and convention of the coordinates. The evolution of the beam size along $z$ axis is measured by moving the collection lens, which is mounted on a 3D translational stage. $\mathbf{b}$, Table of measured FW divergences $\left(\theta_{\mathrm{FW}}\right)$ and SHG divergences $\left(\theta_{\mathrm{SHG}}\right)$ in different confocal systems. c, Linear spin-to-orbital angular momentum conversion efficiencies as a function of the beam divergences of the input light beam. The values of the beam divergences and the conversion efficiencies are experimentally determined. 


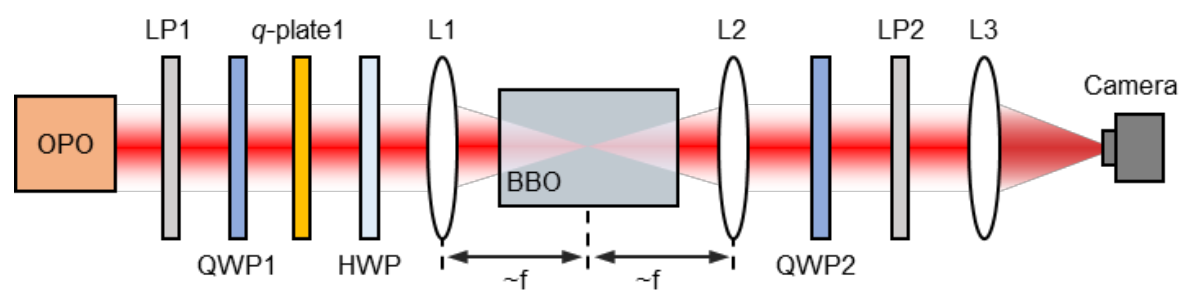

Fig. S10| Optical setup for measuring the linear spin-orbital angular momentum conversion of the $\mathrm{FW}$ in the BBO crystal (paraxial regime). OPO, optical parametric oscillator; LP, linear polarizer; QWP, quarter-wave plate; HWP, half-wave plate; L1 \& L2, objective lenses $(\mathrm{f}=10.5 \mathrm{~mm})$; L3, spherical lens. LP1 \& QWP1 together form a circular polarizer, while QWP2 \& LP2 form a circular analyzer. The $q$-plate1 is used to generate OAM states of FW. The HWP can switch the spin-locked OAM states from $\operatorname{sign}\left(\sigma_{\omega} \ell_{\omega}\right)=-1$ to $\operatorname{sign}\left(\sigma_{\omega} \ell_{\omega}\right)=+1$.

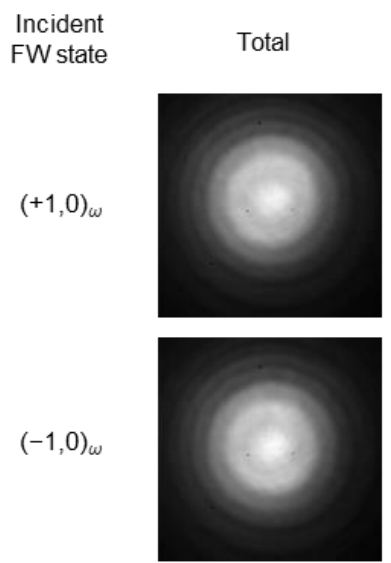

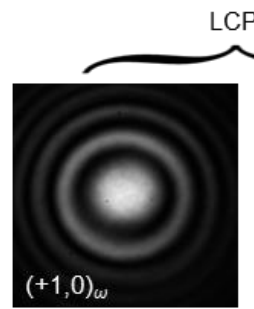

LCP
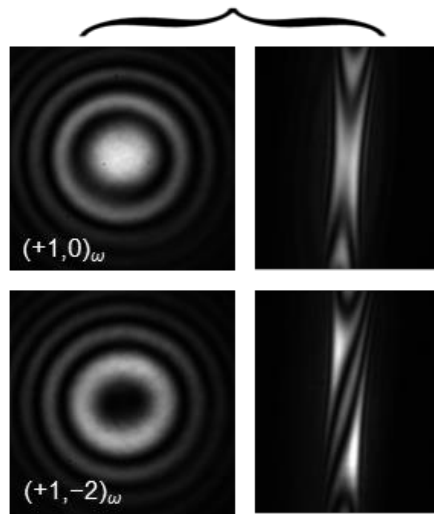
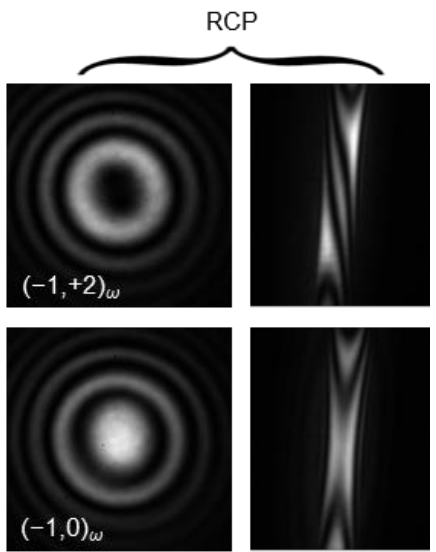

Fig. S11| Spin-orbit content of the output FWs from the BBO crystal for a focused Gaussian FWs (paraxial regime, $\boldsymbol{\ell}_{\boldsymbol{\omega}}=\mathbf{0}$ ). The total intensity profiles as well as the left and right circularly polarized ( $\mathrm{LCP} / \mathrm{RCP}$ ) component profiles are measured (square images). Intensity profiles of LCP or RCP components imaged by cylindrical lens are used to identify the OAM values (rectangle images). The spin-orbital interaction manifests itself in the presence of ring intensity profiles of the contra-polarization components, as well as in the dark fringes of the cylindrical imaged patterns. 


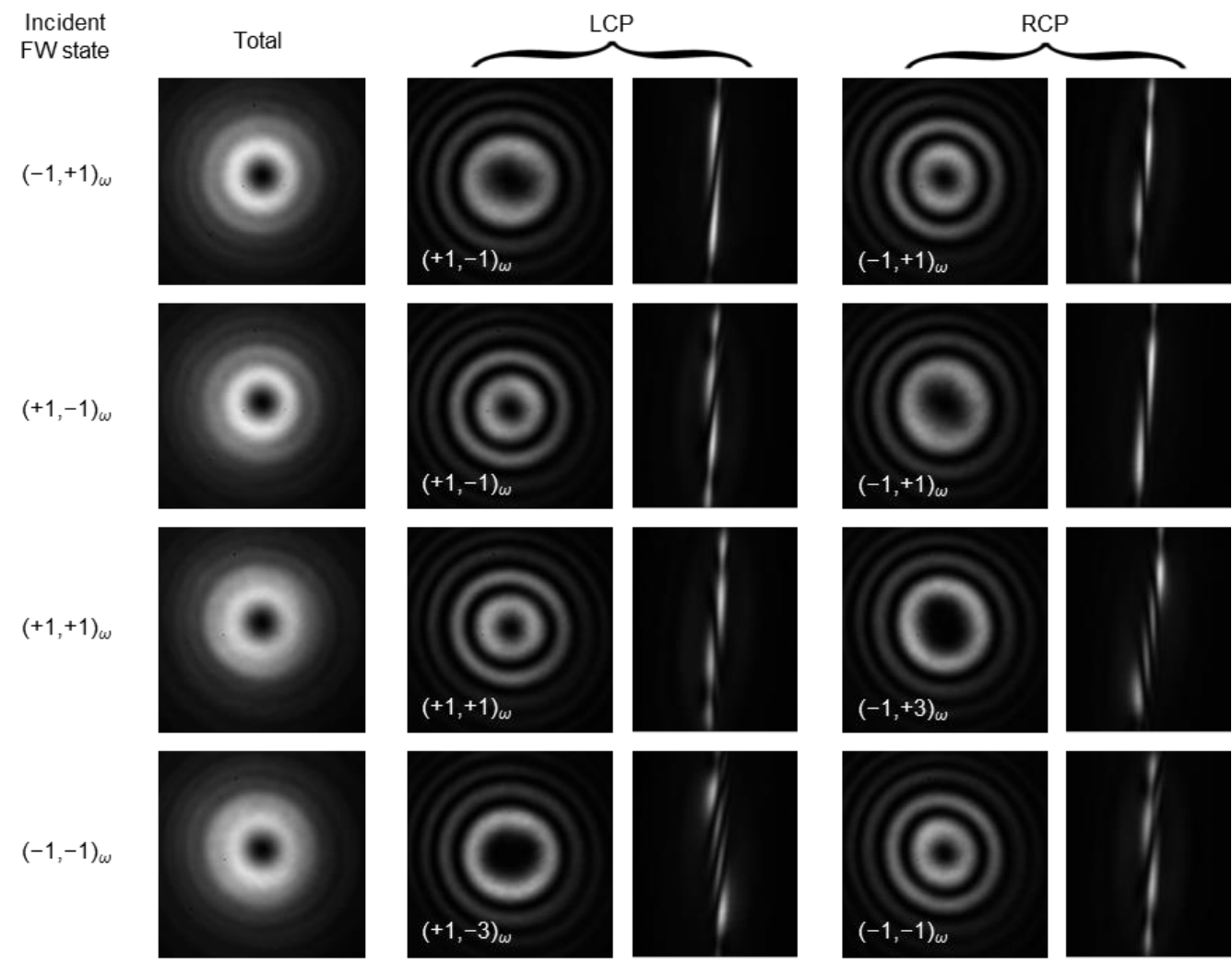

Fig. S12 | Spin-orbit content of the output FWs from the BBO crystal for a focused OAMcarrying FWs (paraxial regime, $\boldsymbol{\ell}_{\omega}= \pm \mathbf{1}$ ). The total intensity profiles as well as the left and right circularly polarized ( $\mathrm{LCP} / \mathrm{RCP}$ ) component profiles are measured (square images). Intensity profiles of LCP or RCP components imaged by cylindrical lens are used to identify the OAM values (rectangle images). 


\section{S4. Spin-Resolved SHG for Focused FW in the Paraxial Regime}

In this section we examine the SHG from the BBO crystal produced by the focused FWs in the paraxial regime. Fig. S13 shows the experiment setup for the measurement of the spin-resolved SHG profiles from the crystal. The results are shown in Figs. S14-S16.

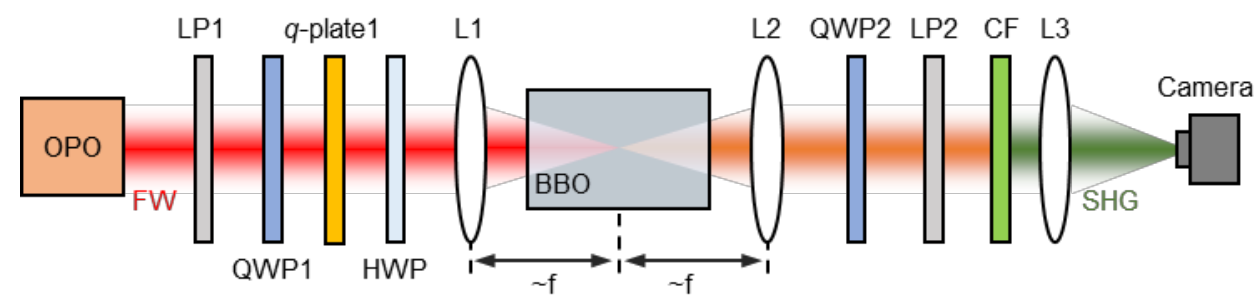

Fig. S13| Optical setup for the measurement of the spin-resolved SHG excited by the focused FWs. OPO, optical parametric oscillator; LP, linear polarizer; QWP, quarter-wave plate; HWP, half-wave plate; L1 \& L2, objective lenses (f=10.5 mm); L3, spherical lens; CF, color filter to filter out the FW at $1070 \mathrm{~nm}$. $q$-plate 1 works at the fundamental frequency and the HWP can switch the spin-locked OAM states after $q$-platel from $\operatorname{sign}\left(\sigma_{\omega} \ell_{\omega}\right)=-1$ to $\operatorname{sign}\left(\sigma_{\omega} \ell_{\omega}\right)=+1$. The LP1 \& QWP1 together form a circular polarizer, while the QWP2 \& LP2 act as a circular analyzer.

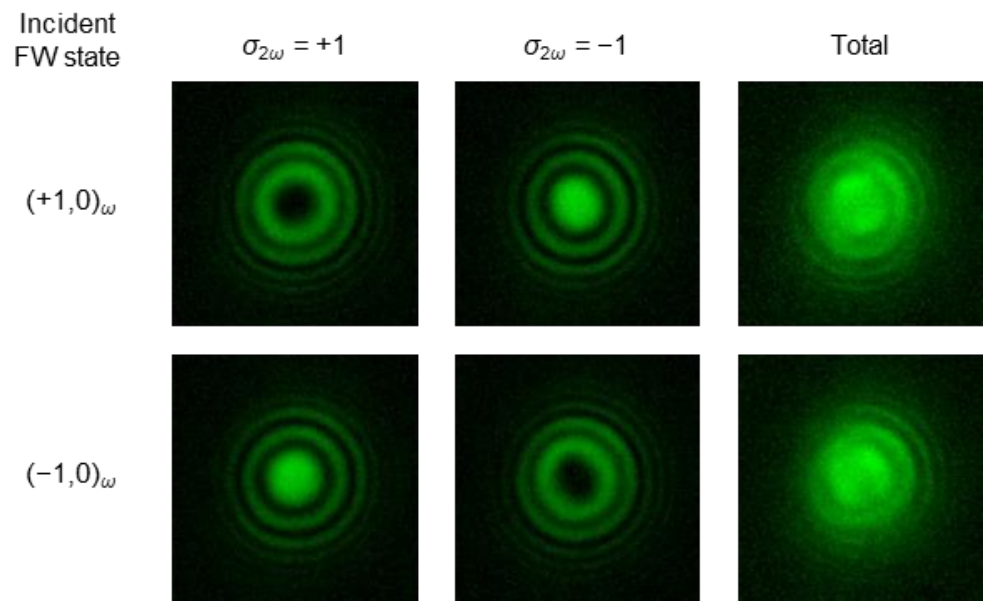

Fig. S14| Spin-resolved intensity profiles of the SHWs generated by the focused Gaussian FWs $\left(\boldsymbol{\ell}_{\boldsymbol{\omega}}=\mathbf{0}\right) \cdot(\sigma, \ell)_{\omega}$ denotes the spin and orbital angular momentum states of the incident FWs, and $\sigma_{2 \omega}$ denotes the spin states of the measured SHWs. 


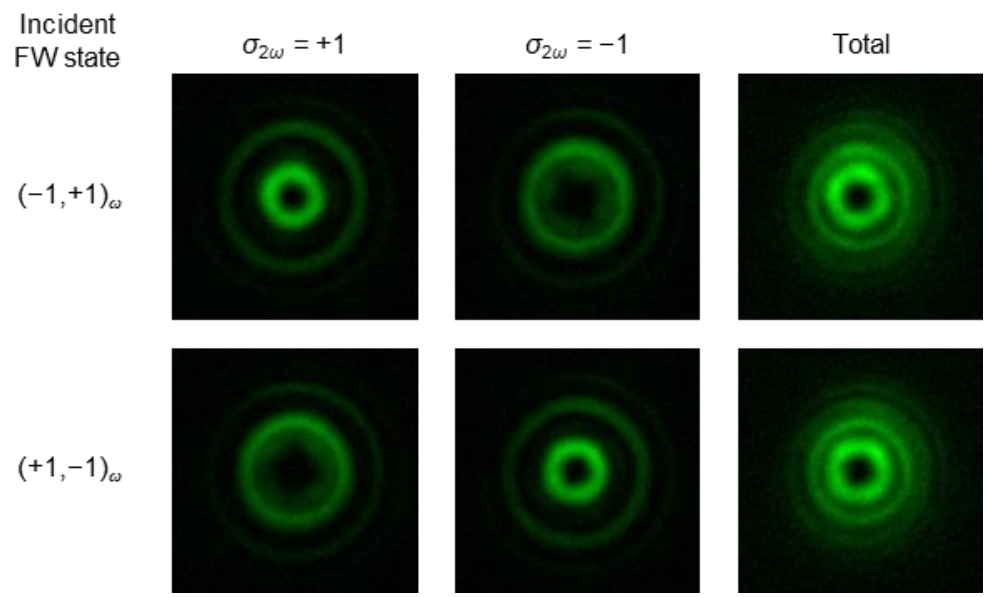

Fig. S15 $\mid$ Spin-resolved intensity profiles of the SHWs generated by focused OAMcarrying FWs $\left(\sigma_{\omega} \ell_{\omega}=-\mathbf{1}\right) . \sigma_{\omega} \ell_{\omega}=-1$ corresponds to no HWP case in Fig. S13.

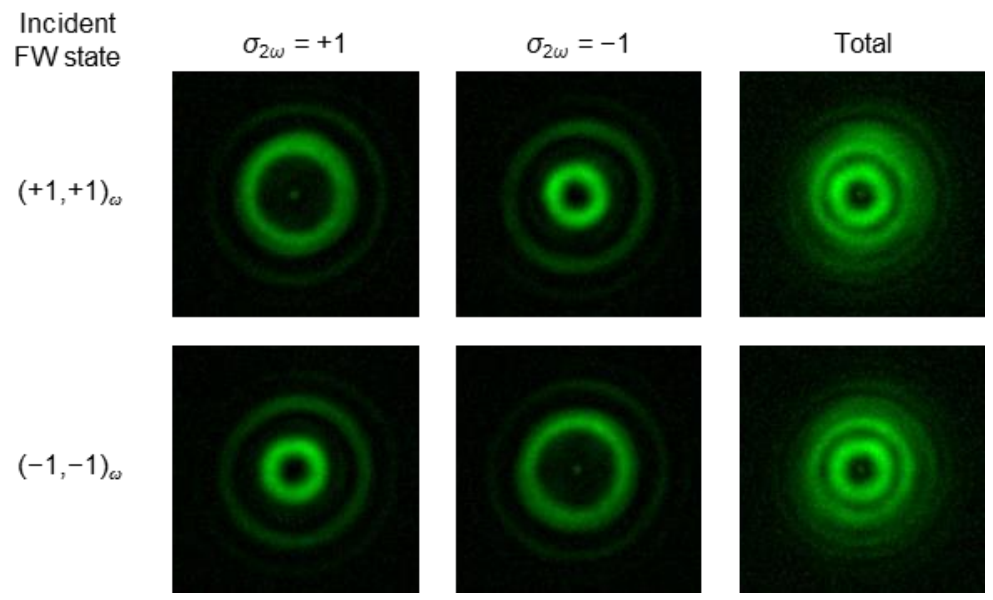

Fig. S16 $\mid$ Spin-resolved intensity profiles of the SHWs generated by focused OAMcarrying FWs $\left(\sigma_{\omega} \ell_{\omega}=+1\right) . \sigma_{\omega} \ell_{\omega}=+1$ corresponds to HWP inserted case in Fig. S13. 


\section{S5. Spin-Orbit State Tomography of SHW for a Focused FW in the Paraxial}

\section{$\underline{\text { Regime }}$}

Here we give the detailed method to unravel the spin-orbit content of the second harmonic field in the BBO crystal. Fig. S17 shows the optical setup for measurement, where two $q$-plates are used ( $q$-plate1 and $q$-plate2). The first $q$-plate 1 works at the fundamental frequency to generate OAM-carrying FW with an azimuthal phase term $\exp ( \pm 2 i q \varphi)$, the second $q$-plate2 works at SHG frequency to impart additional phase term $\exp ( \pm 2 i q \varphi)$ to the selected circularly polarized components of the SHWs. One can recover an on-axis spot when the imparted OAM value $\Delta \ell$ offsets the original carried ones by the SHW. Fig. S18 shows microscopy images of the $q$-plates, which are measured by sandwiching the $q$-plates between two crossed linear polarizers. Fig. S19 shows the complementary results of Fig. 4 in the main text, where the SHWs generated by a focused FW with angular momentum states of $(-1,0)_{\omega}$ and $(-1,+1)_{\omega}$ are presented.

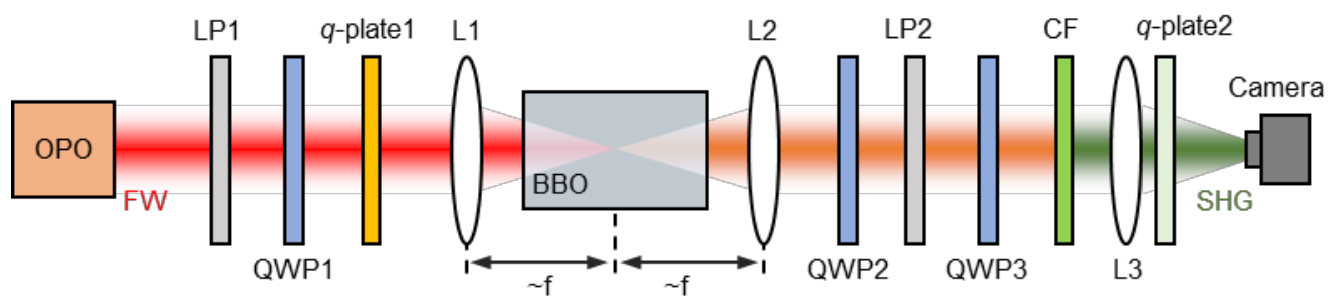

Fig. S17| Spin-orbit state tomography of the angular momentum states of SHWs. A focused FW with angular momentum states of $( \pm 1,0 / \pm 1)_{\omega}$ is used to pump the BBO crystal. OPO, optical parametric oscillator; LP, linear polarizer; QWP, quarter-wave plate; L1 \& L2, objective lenses; L3, spherical lens; CF, color filter to filter out the FW at $1070 \mathrm{~nm}$. $q$-plate 1 works at the fundamental frequency while $q$-plate 2 works at the SHG frequency. QWP1 \& qplate1 control the imparted azimuthal phase profile of the form $\exp ( \pm 2 i q \varphi)$ to the FW. QWP3 $\& q$-plate 2 control the imparted azimuthal phase profile of the form $\exp ( \pm 2 i q \varphi)$ to the selected circular component of the SHW. LP1 \& QWP1 together form a circular polarizer, while QWP2 \& LP2 act as a circular analyzer. 

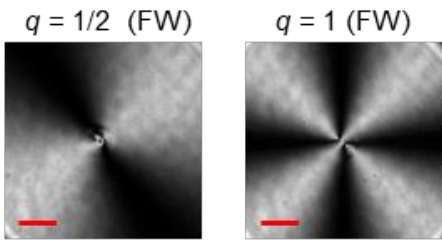

b

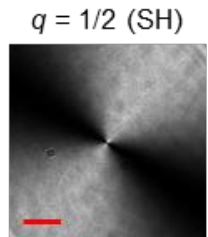

$q=1(\mathrm{SH})$

$q=3 / 2(\mathrm{SH})$
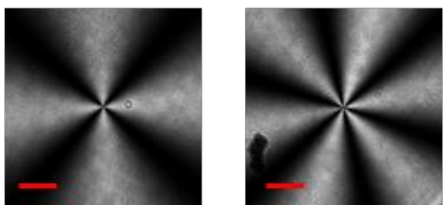

$q=2(\mathrm{SH})$

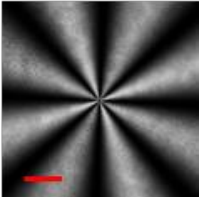

Fig. S18| Microscopy images of the $q$-plates between two crossed linear polarizers. a, $q$ plates for FWs, used as $q$-plate1 in Fig. S17. b, $q$-plates for SHWs, used as $q$-plate2 in Fig. S17. Scale bars: $200 \mu \mathrm{m}$.

a
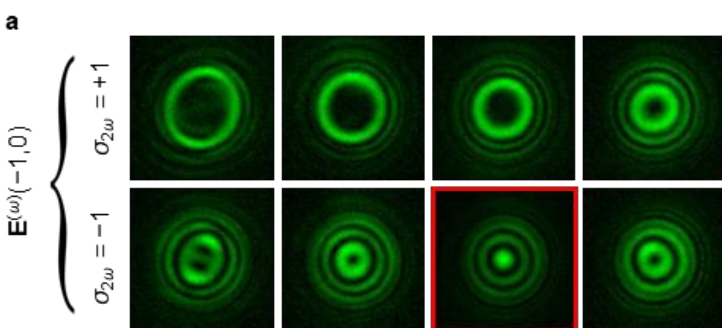

$(-\sigma, 2 \ell)_{2 \omega}$
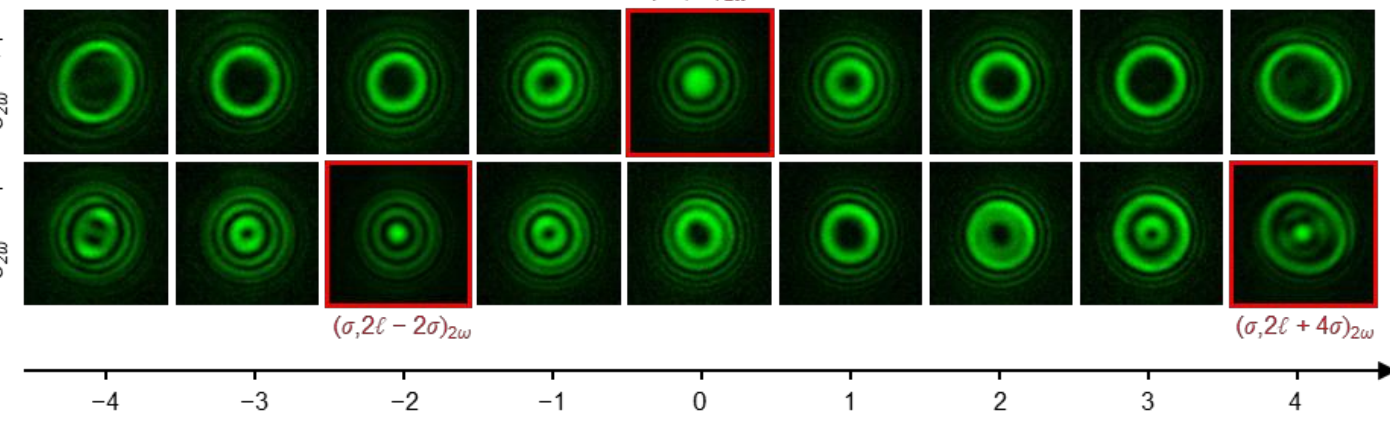

0
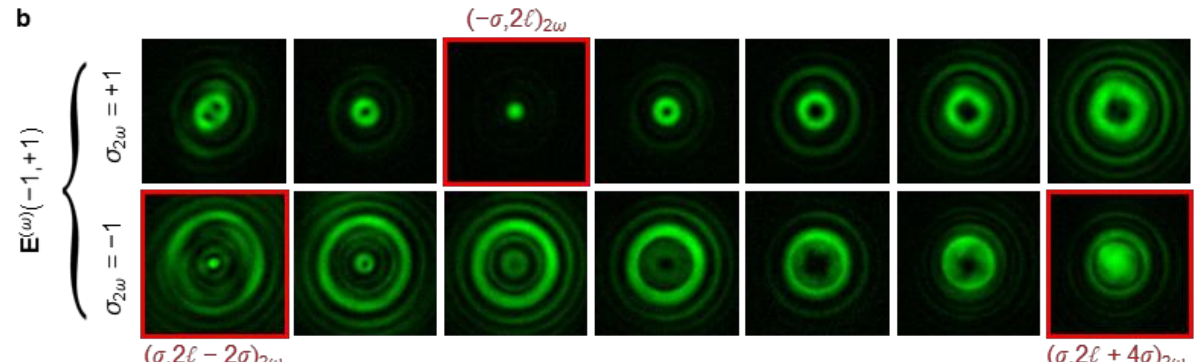

$(\sigma, 2 \ell+4 \sigma)_{2 \omega}$

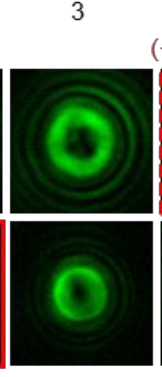

$(\sigma, 2 \ell+4 \sigma)_{2 \omega}$

Fig. S19| Spin-orbit tomography of the second-harmonic field during second harmonic generation along the optical axis of a BBO crystal for weakly focused pumping light. The experimental situation is that presented in Fig. 2. The spin-orbit optical content of the second harmonic field is retrieved by imparting a set of helical phase profiles with topological charge $\Delta \ell$ to each of the circularly polarized field components, by using a set of azimuthally-varying half-wave plates followed by optical Fourier transform performed by a spherical lens. a, The incident fundamental optical state is $(\sigma, \ell)_{\omega}=(-1,0)$. $\mathbf{b}$, The incident fundamental optical state is $(\sigma, \ell)_{\omega}=(-1,+1)$. The fourth predicted state is not observed (see red dashed line box). All the intensity profiles are normalized to the maximal intensity values in each sub-plot. 


\section{S6. Simplified Model and Spin-Orbit Tomography Simulations}

Three-step simplified model for second-harmonic spin-orbit angular momentum cascade

A simplified three-step model is proposed in order to grasp the main features of the combined effects of a nonlinear wave-mixing with spin-orbit coupling when the fundamental wave propagates along the optical axis of a nonlinear crystal with $\mathrm{C}_{3 \mathrm{v}}$ symmetry. This is carried out within a paraxial (non-collimated) framework, where the contribution of the longitudinal field (i.e., along the optical axis of the crystal) can be neglected. The idea is to split the light-matter interaction processes, which occur concurrently, into three distinct steps:

- Step 1: the weakly focused fundamental wave experiences linear spin-orbit coupling as it propagates through the first-half of the crystal, until it reaches the focal plane, where its intensity reaches a maximum.

- Step 2: the second-harmonic takes place in the focal volume of the focused fundamental wave. Since the anisotropic diffraction length associated with the spin-orbit coupling is typically on order of magnitude larger than the longitudinal extent of the focal volume, the second-harmonic generation process is decoupled from spin-orbit interaction at this step.

- Step 3: the generated second-harmonic wave propagates through the second-half of the crystal, during which it experiences linear spin-orbit interaction.

The spin-orbit interaction occurring at step 1 for the fundamental wave is described following previous works on spin-orbit interaction in uniaxial crystals (Refs. 12 and 28). The fundamental wave amplitude distribution in the mid-plane of the crystal for an incident state $(\sigma, \ell)$ is thus expressed as

$$
\mathbf{E}^{(\omega)} \propto u_{\ell}(r) \exp (i \ell \phi)\left\{\left[C_{\omega}(r)+i S_{\omega}(r) e^{2 i \sigma \phi}\right] \mathbf{x}+\sigma\left[i C_{\omega}(r)+S_{\omega}(r) e^{2 i \sigma \phi}\right] \mathbf{y}\right\}
$$

where the complex amplitude $u_{\ell}$ refers here to the Laguerre-Gauss field amplitude (with fundamental radial index $p=0$ and azimuthal index $\ell$ ) used to describe the field at focus (for which the Laguerre-Gauss approximation locally holds) and the functions $C_{\omega}(r)$ and $S_{\omega}(r)$ are cosine and sine functions describing polarization changes as paraxial light propagates into the birefringent crystal. Namely, $C_{\omega}(r)=\cos \left[\Delta_{\omega}(r) / 2\right]$ and $S_{\omega}(r)=\sin \left[\Delta_{\omega}(r) / 2\right]$ where $\Delta_{\omega}(r)=\left(\pi \mathrm{d} n_{\omega} L / \lambda_{\omega}\right)\left(r^{2} / w_{0}^{2}\right) \tan \theta_{0}$ with $\mathrm{d} n_{\omega}$ the birefringence at wavelength $\lambda_{\omega}, w_{0}$ the Gaussian beam waist radius in the focal plane and $\theta_{0}$ the internal Gaussian beam divergence angle.

At step 2, the above fundamental field induces a quadratic polarization according to

$$
P_{i}^{(2 \omega)}=\varepsilon_{0} \sum_{i j k} \chi_{i j k}^{(2)} E_{j}^{(\omega)} E_{k}^{(\omega)}
$$

where $\varepsilon_{0}$ is the vacuum permittivity, $\chi_{i j k}^{(2)}$ is the quadratic susceptibility tensor and the indices $(i, j, k)$ run over the Cartesian coordinates $(x, y, z)$. Recalling the $\mathrm{C}_{3 \mathrm{v}}$ symmetry group of the crystal and assuming the principal mirror-plane perpendicular to the $x$ axis, we have 
$\chi_{y y y}^{(2)}=-\chi_{y x x}^{(2)}=-\chi_{x x y}^{(2)}=-\chi_{x y x}^{(2)}=\chi^{(2)}$. Hence, neglecting the longitudinal field components in the framework of paraxial treatment, one gets

$$
\mathbf{P}^{(2 \omega)}=\varepsilon_{0} \chi^{(2)} \frac{i \sigma}{\sqrt{2}}\left[\left(E_{x}^{(\omega)}+i \sigma E_{y}^{(\omega)}\right)^{2} \mathbf{c}_{\sigma}-\left(E_{x}^{(\omega)}-i \sigma E_{y}^{(\omega)}\right)^{2} \mathbf{c}_{-\sigma}\right]
$$

where the unit vectors $\mathbf{c}_{ \pm \sigma}=(\mathbf{x} \pm i \sigma \mathbf{y}) / \sqrt{2}$ refer to the circular polarization vectors for the spin states $\pm \sigma$. Combining above expression of the fundamental field with the expression of the nonlinear polarization, one gets

$$
\mathbf{P}^{(2 \omega)}(r, \phi) \propto \sigma \varepsilon_{0} \chi^{(2)} u_{\ell}^{2}(r) \exp (2 i \ell \phi)\left[S_{\omega}^{2}(r) e^{4 i \sigma \phi} \mathbf{c}_{\sigma}+C_{\omega}^{2}(r) \mathbf{c}_{-\sigma}\right]
$$

which radiates two cross-circularly polarized second-harmonic components.

At step 3, both circularly polarized components of the generated second-harmonic experience spin-orbit interaction as they propagate through the second half of the crystal. Assuming slowly varying transverse amplitude profiles, the latter components are individually altered in a similar manner then previously described for the fundamental field at step 1 :

$$
\mathbf{c}_{ \pm \sigma} \rightarrow C_{2 \omega}(r) \mathbf{c}_{ \pm \sigma}+i S_{2 \omega}(r) e^{ \pm 2 i \sigma \phi} \mathbf{c}_{\mp \sigma},
$$

where the functions $C_{2 \omega}(r)$ and $S_{2 \omega}(r)$ are now specified for the harmonic wavelength. This results into two spin-orbit states for each of the spin-orbit states generated at step 2, hence four in total. Namely,

$$
\{(-\sigma, 2 \ell) ;(\sigma, 2 \ell-2 \sigma) ;(\sigma, 2 \ell+4 \sigma) ;(-\sigma, 2 \ell+6 \sigma)\},
$$

and the whole process in summarized in Fig. 3 of the main text. Of course, this description is simplified as it does not take into account the integral aspect of the process since all three steps mentioned here all occur simultaneously along the propagation of light through the nonlinear crystal. Still, this is enough to capture the essence of the reported effects, which is well supported by our observations.

Above three-step model allows expressing the second-harmonic field after the crystal. In a plane where the fundamental wave radius waist of the total intensity is $W$, with $\rho=r / W$, we thus eventually have

$$
\begin{aligned}
E^{(2 \omega)}(\rho, \phi) \propto & \sigma \chi^{(2)} \rho^{2|\ell|} e^{-2 \rho^{2}+2 i \ell \phi} \\
& \times\left\{\left[S_{\omega}^{2}(\rho) C_{2 \omega}(\rho) e^{4 i \sigma \phi}+i C_{\omega}^{2}(\rho) S_{2 \omega}(\rho) e^{-2 i \sigma \phi}\right] \mathbf{c}_{\sigma} .\right. \\
& \left.+\left[i S_{\omega}^{2}(\rho) S_{2 \omega}(\rho) e^{6 i \sigma \phi}+C_{\omega}^{2}(\rho) C_{2 \omega}(\rho)\right] \mathbf{c}_{-\sigma}\right\}
\end{aligned}
$$

Above expression allows simulating the recorder images when implementing the spinorbit tomography, see Fig. 4 of the main text. This is done by applying to the above field a helical phase mask $\exp (i \Delta \ell \varphi)$, with $\Delta \ell$ integer and $-4 \leq \Delta \ell \leq+4$ in the experiments, and then calculating the squared Fourier transform, which mimics the intensity pattern recorded in the focal plane of a lens, see Fig. S17:

$$
I^{(2 \omega)}=\left|\mathcal{F}\left[E^{(2 \omega)}(\rho, \phi) e^{i \Delta \ell \phi}\right]\right|^{2}
$$

where $\mathcal{F}$ refers to the two-dimensional Fourier transform. The simulation results are shown in 
Figs. S20 and S21 for incident fundamental wave states $( \pm 1,0)_{\omega},(-1,+1)_{\omega}$ and $(+1,-1)_{\omega}$, respectively. Noteworthy, as we observe in the experiments (almost) axisymmetric intensity profiles, the above simulations are made by introducing a decoherence term between the two contributions of each circularly polarized components. The physical reason for the nonobservation of such interferences remains to be clarified.

Obviously, the present approach is too simplistic and does not aim at being quantitative. Still, it allows capturing the essential aspects of the combined effects of optical nonlinearity and spin-orbit coupling. There is plenty of room for further improvements, in particular taking into account spatially distributed second-harmonic generation along the propagation direction as well as the transverse integral overlap between the fundamental and the second-harmonic wave.
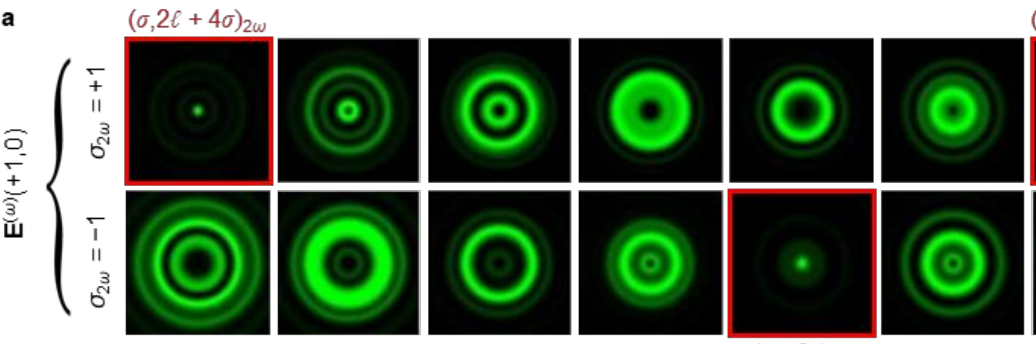

$(\sigma, 2 \ell-2 \sigma)_{2 \omega}$
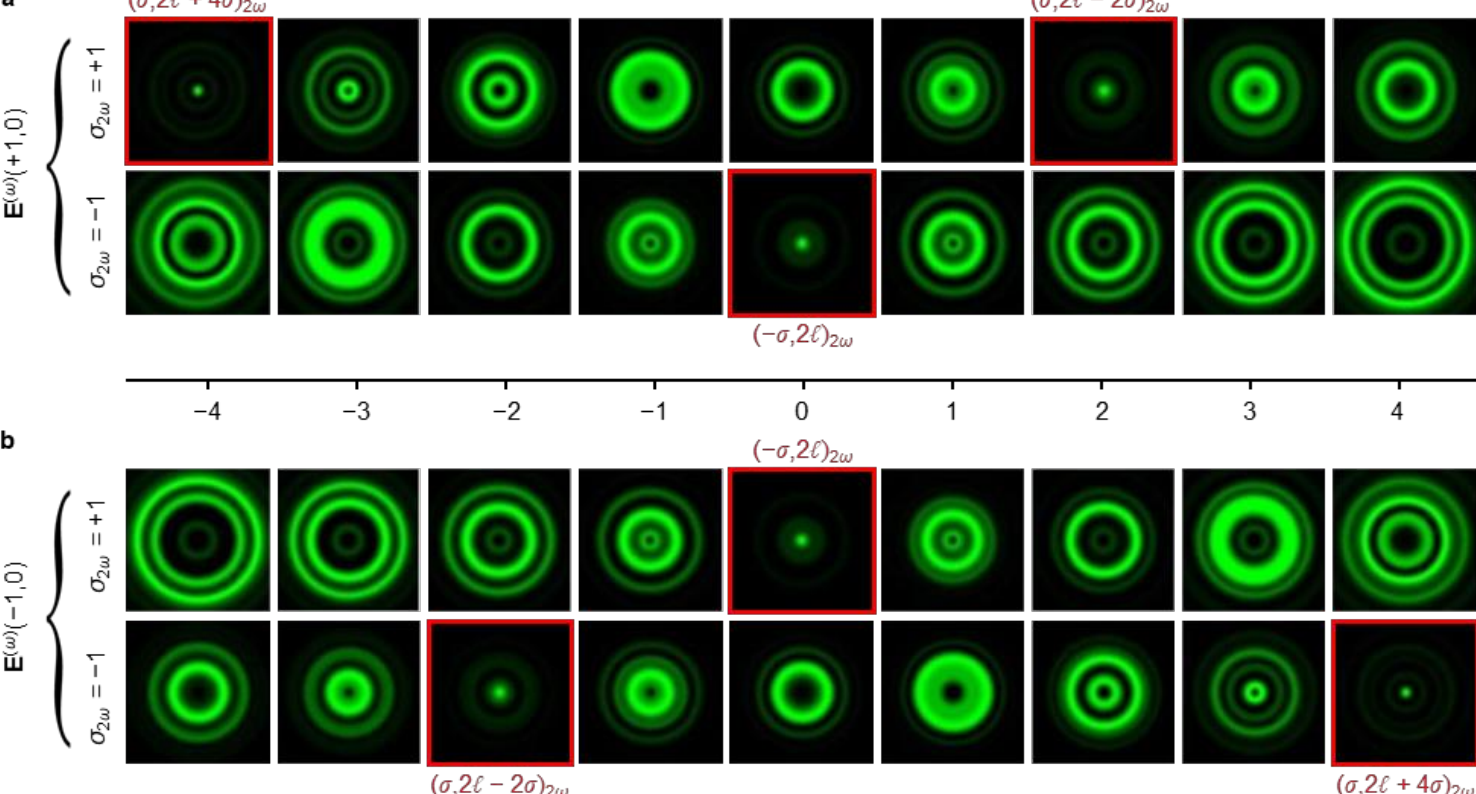

$(-\sigma, 2 \ell)_{2 \omega}$
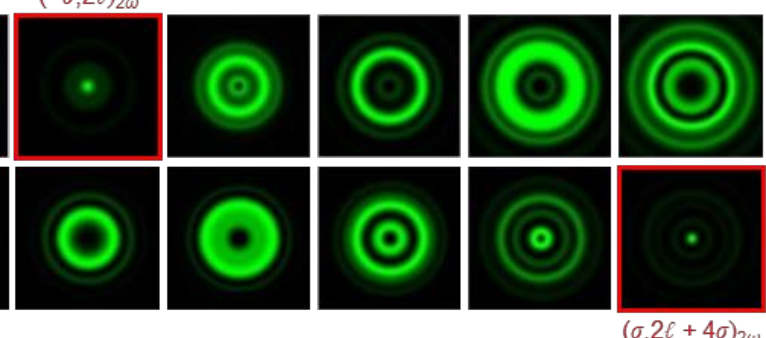

Fig. S20| Simulated results for the Fourier projective measurements. The secondharmonic fields in the BBO crystal are generated by using the focused FWs with angular momentum states of $( \pm 1,0)_{\omega}$. 

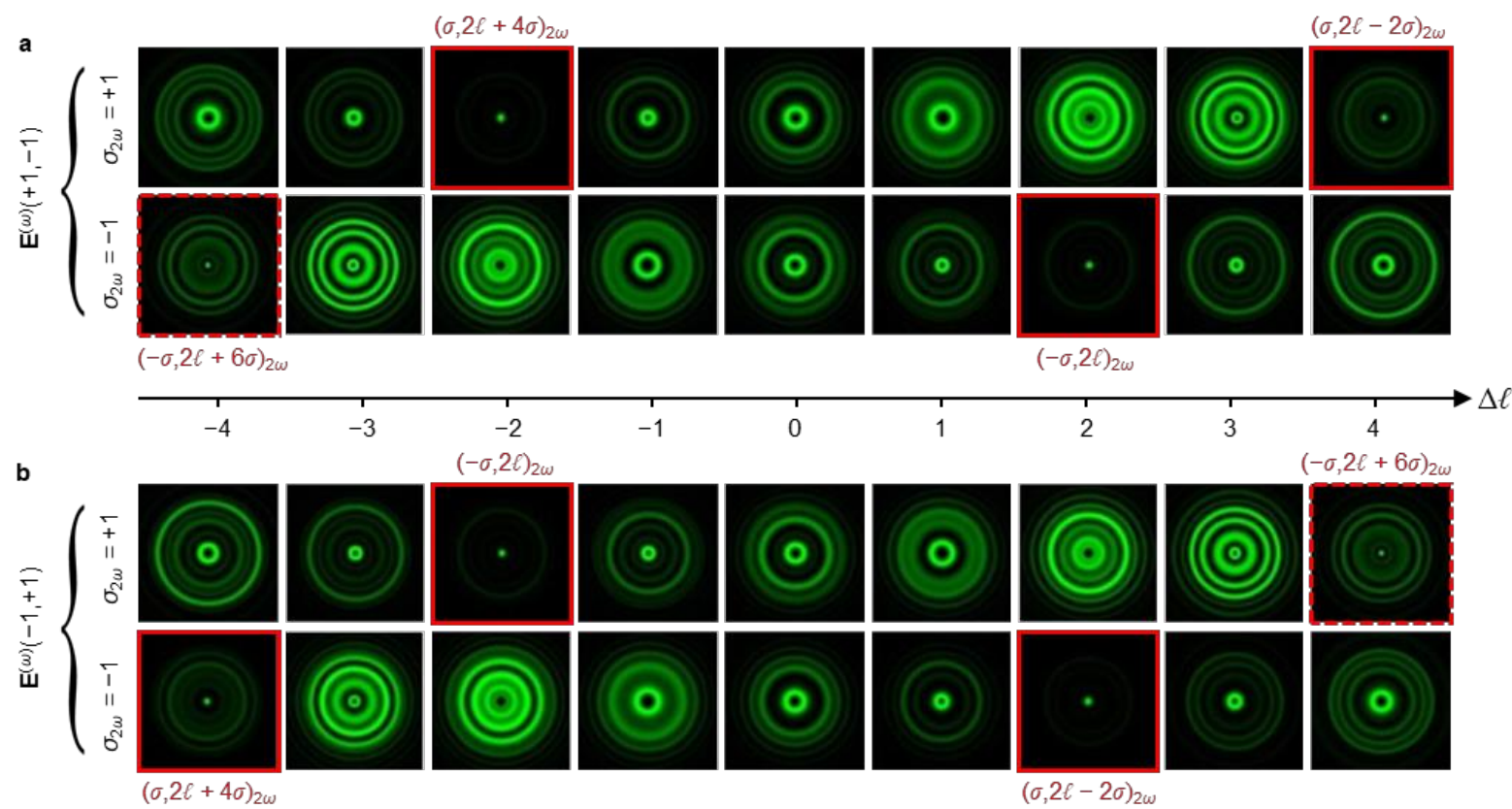

Fig. S21| Simulated results for the Fourier projective measurements. The secondharmonic fields in the BBO crystal are generated by using the focused FWs with angular momentum states of $(-1,+1) \omega$ and $(+1,-1) \omega$. 


\section{$\underline{\text { S7. Angular Momentum States of } n^{\text {th }} \text { Harmonic Generation Waves }}$}

Assuming we have a circularly polarized FW propagating along the optical axis of a crystal with $m$-fold rotational symmetry, the allowed order $n$ of the harmonic generation is determined by $n=p m \pm 1$, where $p$ is a positive integer, \pm corresponds to the harmonic generation waves with the same and opposite circular polarization to that of the FWs (Ref. 30). When the fundamental wave has both spin and orbital angular momentum state $(\sigma, \ell)_{\omega}$, we then have two states $(\sigma, \ell)_{\omega}$ and $(-\sigma, \ell+2 \sigma)_{\omega}$ after the spin-orbit process in the nonlinear optical crystal.

Next, we start to consider the high harmonic generations (HHG) in the nonlinear optical crystal. From both the symmetry selection rules in nonlinear optics and the conservation law of OAM, we can predict that for the $n^{\text {th }}$ order HHG wave with spin state the same as that of the FW, the following angular momentum states: $[\sigma, n \ell]_{n \omega}$ and $[-\sigma, n(\ell+2 \sigma)]_{n \omega}$ can be generated. For HHG waves with opposite circular polarizations to that of the $\mathrm{FW}$, the angular momentum states are $[-\sigma, n \ell]_{n \omega}$ and $[\sigma, n(\ell+2 \sigma)]_{n \omega}$, respectively. For the FW at state of $(\sigma, \ell)_{\omega}$, the OAM states of the HHG waves with the opposite and same circular polarizations are summarized in Table S1 and Table S2.

\begin{tabular}{|c|c|c|c|c|c|c|c|c|}
\hline Spin $\boldsymbol{\sigma}_{\boldsymbol{\omega}}$ to $-\boldsymbol{\sigma}_{\boldsymbol{n} \boldsymbol{\omega}}$ & $\boldsymbol{n = 2}$ & $\boldsymbol{n = 3}$ & $\boldsymbol{n = 4}$ & $\boldsymbol{n = 5}$ & $\mathbf{n = 6}$ & $\boldsymbol{n = 7}$ & $\boldsymbol{n = 8}$ & $\boldsymbol{n = 9}$ \\
\hline $\mathrm{C}_{1}$ & $2 \ell$ & $3 \ell$ & $4 \ell$ & $5 \ell$ & $6 \ell$ & $7 \ell$ & $8 \ell$ & $9 \ell$ \\
\hline $\mathrm{C}_{2}$ & $\mathrm{x}$ & $3 \ell$ & $\mathrm{x}$ & $5 \ell$ & $\mathrm{x}$ & $7 \ell$ & $\mathrm{x}$ & $9 \ell$ \\
\hline $\mathrm{C}_{3}$ & $2 \ell$ & $\mathrm{x}$ & $\mathrm{x}$ & $5 \ell$ & $\mathrm{x}$ & $\mathrm{x}$ & $8 \ell$ & $\mathrm{x}$ \\
\hline $\mathrm{C}_{4}$ & $\mathrm{x}$ & $3 \ell$ & $\mathrm{x}$ & $\mathrm{x}$ & $\mathrm{x}$ & $7 \ell$ & $\mathrm{x}$ & $\mathrm{x}$ \\
\hline $\mathrm{C}_{6}$ & $\mathrm{x}$ & $\mathrm{x}$ & $\mathrm{x}$ & $5 \ell$ & $\mathrm{x}$ & $\mathrm{x}$ & $\mathrm{x}$ & $\mathrm{x}$ \\
\hline
\end{tabular}

Table S1 | Angular momentum states of the harmonic generation waves with opposite circular polarization to that of the fundamental wave with $(\sigma, \ell)_{\omega} . n=2-9$ are the orders of harmonic generations, $\mathrm{C}_{1}-\mathrm{C}_{6}$ represent the one-fold, two-fold, three-fold, four-fold and six-fold rotational symmetries of the nonlinear crystals. Here the allowed states are $(-\sigma, n \ell)_{n \omega}$, and ' $\mathrm{x}$ ' means the related harmonic generation is forbidden.

\begin{tabular}{|c|c|c|c|c|c|c|c|c|}
\hline Spin $\boldsymbol{\sigma}_{\boldsymbol{\omega}}$ to $\boldsymbol{\sigma}_{\boldsymbol{n} \boldsymbol{\omega}}$ & $\boldsymbol{n = 2}$ & $\boldsymbol{n = 3}$ & $\boldsymbol{n = 4}$ & $\boldsymbol{n = 5}$ & $\boldsymbol{n = 6}$ & $\boldsymbol{n = 7}$ & $\boldsymbol{n = 8}$ & $\boldsymbol{n = 9}$ \\
\hline $\mathrm{C}_{1}$ & $2 \ell$ & $3 \ell$ & $4 \ell$ & $5 \ell$ & $6 \ell$ & $7 \ell$ & $8 \ell$ & $9 \ell$ \\
\hline $\mathrm{C}_{2}$ & $\mathrm{x}$ & $3 \ell$ & $\mathrm{x}$ & $5 \ell$ & $\mathrm{x}$ & $7 \ell$ & $\mathrm{x}$ & $9 \ell$ \\
\hline $\mathrm{C}_{3}$ & $\mathrm{x}$ & $\mathrm{x}$ & $4 \ell$ & $\mathrm{x}$ & $\mathrm{x}$ & $7 \ell$ & $\mathrm{x}$ & $\mathrm{x}$ \\
\hline $\mathrm{C}_{4}$ & $\mathrm{x}$ & $\mathrm{x}$ & $\mathrm{x}$ & $5 \ell$ & $\mathrm{x}$ & $\mathrm{x}$ & $\mathrm{x}$ & $9 \ell$ \\
\hline $\mathrm{C}_{6}$ & $\mathrm{x}$ & $\mathrm{x}$ & $\mathrm{x}$ & $\mathrm{x}$ & $\mathrm{x}$ & $7 \ell$ & $\mathrm{x}$ & $\mathrm{x}$ \\
\hline
\end{tabular}

Table S2 | Angular momentum states of harmonic generation waves with same circular polarization as that of the fundamental wave with $(\sigma, \ell)_{\omega}$. Here the allowed states are $(\sigma$, $n \ell)_{n \omega}$, and ' $\mathrm{x}$ ' means the related harmonic generation is forbidden.

For the FW at state of $(-\sigma, \ell+2 \sigma)_{\omega}$, the OAM states of the HHG waves with the opposite 
and same circular polarizations are summarized in Table S3 and Table S4.

\begin{tabular}{|c|c|c|c|c|c|c|c|c|}
\hline Spin $-\boldsymbol{\sigma}_{\boldsymbol{\omega}}$ to $\boldsymbol{\sigma}_{\boldsymbol{n} \boldsymbol{\omega}}$ & $\boldsymbol{n = 2}$ & $\boldsymbol{n = 3}$ & $\boldsymbol{n = 4}$ & $\boldsymbol{n = 5}$ & $\boldsymbol{n = 6}$ & $\boldsymbol{n = 7}$ & $\boldsymbol{n = 8}$ & $\boldsymbol{n = 9}$ \\
\hline $\mathrm{C}_{1}$ & $2(\ell+2 \sigma)$ & $3(\ell+2 \sigma)$ & $4(\ell+2 \sigma)$ & $5(\ell+2 \sigma)$ & $6(\ell+2 \sigma)$ & $7(\ell+2 \sigma)$ & $8(\ell+2 \sigma)$ & $9(\ell+2 \sigma)$ \\
\hline $\mathrm{C}_{2}$ & $\mathrm{x}$ & $3(\ell+2 \sigma)$ & $\mathrm{x}$ & $5(\ell+2 \sigma)$ & $\mathrm{x}$ & $7(\ell+2 \sigma)$ & $\mathrm{x}$ & $9(\ell+2 \sigma)$ \\
\hline $\mathrm{C}_{3}$ & $2(\ell+2 \sigma)$ & $\mathrm{x}$ & $\mathrm{x}$ & $5(\ell+2 \sigma)$ & $\mathrm{x}$ & $\mathrm{x}$ & $8(\ell+2 \sigma)$ & $\mathrm{x}$ \\
\hline $\mathrm{C}_{4}$ & $\mathrm{x}$ & $3(\ell+2 \sigma)$ & $\mathrm{x}$ & $\mathrm{x}$ & $\mathrm{x}$ & $7(\ell+2 \sigma)$ & $\mathrm{x}$ & $\mathrm{x}$ \\
\hline $\mathrm{C}_{6}$ & $\mathrm{x}$ & $\mathrm{x}$ & $\mathrm{x}$ & $5(\ell+2 \sigma)$ & $\mathrm{x}$ & $\mathrm{x}$ & $\mathrm{x}$ & $\mathrm{x}$ \\
\hline
\end{tabular}

Table S3 | Angular momentum states of harmonic generation waves with opposite circular polarization to that of the fundamental wave $(-\sigma, \boldsymbol{\ell}+\mathbf{2} \sigma)_{\boldsymbol{\omega}}$. Here the allowed states are $[\sigma$, $n(\ell+2 \sigma)]_{n \omega}$, and ' $\mathrm{x}$ ' means the related harmonic generation is forbidden.

\begin{tabular}{|c|c|c|c|c|c|c|c|c|}
\hline Spin $-\boldsymbol{\sigma}_{\boldsymbol{\omega}}$ to $-\boldsymbol{\sigma}_{\boldsymbol{n} \boldsymbol{\omega}}$ & $\boldsymbol{n = 2}$ & $\boldsymbol{n = 3}$ & $\boldsymbol{n = 4}$ & $\boldsymbol{n = 5}$ & $\boldsymbol{n = 6}$ & $\boldsymbol{n = 7}$ & $\boldsymbol{n = 8}$ & $\boldsymbol{n}=\mathbf{9}$ \\
\hline $\mathrm{C}_{1}$ & $2(\ell+2 \sigma)$ & $3(\ell+2 \sigma)$ & $4(\ell+2 \sigma)$ & $5(\ell+2 \sigma)$ & $6(\ell+2 \sigma)$ & $7(\ell+2 \sigma)$ & $8(\ell+2 \sigma)$ & $9(\ell+2 \sigma)$ \\
\hline $\mathrm{C}_{2}$ & $\mathrm{x}$ & $3(\ell+2 \sigma)$ & $\mathrm{x}$ & $5(\ell+2 \sigma)$ & $\mathrm{x}$ & $7(\ell+2 \sigma)$ & $\mathrm{x}$ & $9(\ell+2 \sigma)$ \\
\hline $\mathrm{C}_{3}$ & $\mathrm{x}$ & $\mathrm{x}$ & $4(\ell+2 \sigma)$ & $\mathrm{x}$ & $\mathrm{x}$ & $7(\ell+2 \sigma)$ & $\mathrm{x}$ & $\mathrm{x}$ \\
\hline $\mathrm{C}_{4}$ & $\mathrm{x}$ & $\mathrm{x}$ & $\mathrm{x}$ & $5(\ell+2 \sigma)$ & $\mathrm{x}$ & $\mathrm{x}$ & $\mathrm{x}$ & $9(\ell+2 \sigma)$ \\
\hline $\mathrm{C}_{6}$ & $\mathrm{x}$ & $\mathrm{x}$ & $\mathrm{x}$ & $\mathrm{x}$ & $\mathrm{x}$ & $7(\ell+2 \sigma)$ & $\mathrm{x}$ & $\mathrm{x}$ \\
\hline
\end{tabular}

Table S4 | Angular momentum states of harmonic generation waves with same circular polarization as that of the fundamental wave $(-\sigma, \boldsymbol{\ell}+\mathbf{2} \sigma)_{\omega}$. Here the allowed states are $[-\sigma$, $n(\ell+2 \sigma)]_{n \omega}$, and ' $\mathrm{x}$ ' means the related harmonic generation is forbidden.

The states shown in Table S1-S4 can further lead new angular momentums states through to spin-orbit cascaded processes of the harmonic generation waves. To be specific, states $[\sigma$, $n \ell]_{n \omega}$ and $[-\sigma, n(\ell+2 \sigma)]_{n \omega}$ can lead to $[-\sigma, n \ell+2 \sigma]_{n \omega}$ and $[\sigma, n \ell+2(n-1) \sigma]_{n \omega}$, while states $[-\sigma$, $n \ell]_{n \omega}$ and $[\sigma, n(\ell+2 \sigma)]_{n \omega}$ lead to $[\sigma, n \ell-2 \sigma]_{n \omega}$ and $[-\sigma, n \ell+2(n+1) \sigma]_{n \omega}$. These states are summarized in Table S5-S8.

\begin{tabular}{|c|c|c|c|c|c|c|c|c|}
\hline Spin $\boldsymbol{\sigma}_{\boldsymbol{\omega}}$ to $\boldsymbol{\sigma}_{\boldsymbol{n} \boldsymbol{\omega}}$ & $\boldsymbol{n = 2}$ & $\boldsymbol{n = 3}$ & $\boldsymbol{n}=\mathbf{4}$ & $\boldsymbol{n}=\mathbf{5}$ & $\boldsymbol{n = 6}$ & $\boldsymbol{n = 7}$ & $\boldsymbol{n = 8}$ & $\boldsymbol{n}=\mathbf{9}$ \\
\hline $\mathrm{C}_{1}$ & $2 \ell-2 \sigma$ & $3 \ell-2 \sigma$ & $4 \ell-2 \sigma$ & $5 \ell-2 \sigma$ & $6 \ell-2 \sigma$ & $7 \ell-2 \sigma$ & $8 \ell-2 \sigma$ & $9 \ell-2 \sigma$ \\
\hline $\mathrm{C}_{2}$ & $\mathrm{x}$ & $3 \ell-2 \sigma$ & $\mathrm{x}$ & $5 \ell-2 \sigma$ & $\mathrm{x}$ & $7 \ell-2 \sigma$ & $\mathrm{x}$ & $9 \ell-2 \sigma$ \\
\hline $\mathrm{C}_{3}$ & $2 \ell-2 \sigma$ & $\mathrm{x}$ & $\mathrm{x}$ & $5 \ell-2 \sigma$ & $\mathrm{x}$ & $\mathrm{x}$ & $8 \ell$ & $\mathrm{x}$ \\
\hline $\mathrm{C}_{4}$ & $\mathrm{x}$ & $3 \ell-2 \sigma$ & $\mathrm{x}$ & $\mathrm{x}$ & $\mathrm{x}$ & $7 \ell-2 \sigma$ & $\mathrm{x}$ & $\mathrm{x}$ \\
\hline $\mathrm{C}_{6}$ & $\mathrm{x}$ & $\mathrm{x}$ & $\mathrm{x}$ & $5 \ell-2 \sigma$ & $\mathrm{x}$ & $\mathrm{x}$ & $\mathrm{x}$ & $\mathrm{x}$ \\
\hline
\end{tabular}

Table S5 | Angular momentum states of the harmonic generation waves with opposite circular polarization to that of the fundamental wave with $(\sigma, \ell)_{\omega}$, and further undergo a spin-orbit cascade process. Here the allowed states are $[\sigma, n \ell-2 \sigma]_{n \omega}$, and ' $\mathrm{x}$ ' means the related harmonic generation is forbidden. This table follows Table S1. 


\begin{tabular}{|c|c|c|c|c|c|c|c|c|}
\hline Spin $\boldsymbol{\sigma}_{\boldsymbol{\omega}}$ to $-\boldsymbol{\sigma}_{\boldsymbol{n} \boldsymbol{\omega}}$ & $\boldsymbol{n = 2}$ & $\boldsymbol{n = 3}$ & $\boldsymbol{n = 4}$ & $\boldsymbol{n = 5}$ & $\boldsymbol{n = 6}$ & $\boldsymbol{n = 7}$ & $\boldsymbol{n = 8}$ & $\boldsymbol{n = 9}$ \\
\hline $\mathrm{C}_{1}$ & $2 \ell+2 \sigma$ & $3 \ell+2 \sigma$ & $4 \ell+2 \sigma$ & $5 \ell+2 \sigma$ & $6 \ell+2 \sigma$ & $7 \ell+2 \sigma$ & $8 \ell+2 \sigma$ & $9 \ell+2 \sigma$ \\
\hline $\mathrm{C}_{2}$ & $\mathrm{x}$ & $3 \ell+2 \sigma$ & $\mathrm{x}$ & $5 \ell+2 \sigma$ & $\mathrm{x}$ & $7 \ell+2 \sigma$ & $\mathrm{x}$ & $9 \ell+2 \sigma$ \\
\hline $\mathrm{C}_{3}$ & $\mathrm{x}$ & $\mathrm{x}$ & $4 \ell+2 \sigma$ & $\mathrm{x}$ & $\mathrm{x}$ & $7 \ell+2 \sigma$ & $\mathrm{x}$ & $\mathrm{x}$ \\
\hline $\mathrm{C}_{4}$ & $\mathrm{x}$ & $\mathrm{x}$ & $\mathrm{x}$ & $5 \ell+2 \sigma$ & $\mathrm{x}$ & $\mathrm{x}$ & $\mathrm{x}$ & $9 \ell+2 \sigma$ \\
\hline $\mathrm{C}_{6}$ & $\mathrm{x}$ & $\mathrm{x}$ & $\mathrm{x}$ & $\mathrm{x}$ & $\mathrm{x}$ & $7 \ell+2 \sigma$ & $\mathrm{x}$ & $\mathrm{x}$ \\
\hline
\end{tabular}

Table S6 | Angular momentum states of harmonic generation waves with same circular polarization as that of the fundamental wave with $(\sigma, \ell)_{\omega}$, and further undergo a spinorbit cascade process. Here the allowed states are $[-\sigma, n \ell+2 \sigma]_{n \omega}$, and ' $\mathrm{x}$ ' means the related harmonic generation is forbidden. This table follows Table S2.

\begin{tabular}{|c|c|c|c|c|c|c|c|c|}
\hline Spin $-\boldsymbol{\sigma}_{\boldsymbol{\omega}}$ to $-\boldsymbol{\sigma}_{\boldsymbol{n} \boldsymbol{\omega}}$ & $\boldsymbol{n = 2}$ & $\boldsymbol{n = 3}$ & $\boldsymbol{n = 4}$ & $\boldsymbol{n = 5}$ & $\boldsymbol{n = 6}$ & $\boldsymbol{n = 7}$ & $\boldsymbol{n = 8}$ & $\boldsymbol{n = 9}$ \\
\hline $\mathrm{C}_{1}$ & $2 \ell+6 \sigma$ & $3 \ell+8 \sigma$ & $4 \ell+10 \sigma$ & $5 \ell+12 \sigma$ & $6 \ell+14 \sigma$ & $7 \ell+16 \sigma$ & $8 \ell+18 \sigma$ & $9 \ell+20 \sigma$ \\
\hline $\mathrm{C}_{2}$ & $\mathrm{x}$ & $3 \ell+8 \sigma$ & $\mathrm{x}$ & $5 \ell+12 \sigma$ & $\mathrm{x}$ & $7 \ell+16 \sigma$ & $\mathrm{x}$ & $9 \ell+20 \sigma$ \\
\hline $\mathrm{C}_{3}$ & $2 \ell+6 \sigma$ & $\mathrm{x}$ & $\mathrm{x}$ & $5 \ell+12 \sigma$ & $\mathrm{x}$ & $\mathrm{x}$ & $8 \ell+18 \sigma$ & $\mathrm{x}$ \\
\hline $\mathrm{C}_{4}$ & $\mathrm{x}$ & $3 \ell+8 \sigma$ & $\mathrm{x}$ & $\mathrm{x}$ & $\mathrm{x}$ & $7 \ell+16 \sigma$ & $\mathrm{x}$ & $\mathrm{x}$ \\
\hline $\mathrm{C}_{6}$ & $\mathrm{x}$ & $\mathrm{x}$ & $\mathrm{x}$ & $5 \ell+12 \sigma$ & $\mathrm{x}$ & $\mathrm{x}$ & $\mathrm{x}$ & $\mathrm{x}$ \\
\hline
\end{tabular}

Table S7 | Angular momentum states of harmonic generation waves with opposite circular polarization to that of the fundamental wave $(-\sigma, \ell+2 \sigma)_{\omega}$, and further undergo a spinorbit cascade process. Here the allowed states are $[-\sigma, n \ell+2(n+1) \sigma]_{n \omega}$, and ' $\mathrm{x}$ ' means the related harmonic generation is forbidden. This table follows Table S3.

\begin{tabular}{|c|c|c|c|c|c|c|c|c|}
\hline Spin $-\boldsymbol{\sigma}_{\boldsymbol{\omega}}$ to $\boldsymbol{\sigma}_{\boldsymbol{n} \boldsymbol{\omega}}$ & $\boldsymbol{n = 2}$ & $\boldsymbol{n = 3}$ & $\boldsymbol{n = 4}$ & $\boldsymbol{n = 5}$ & $\boldsymbol{n = 6}$ & $\boldsymbol{n}=\mathbf{7}$ & $\boldsymbol{n = 8}$ & $\boldsymbol{n = 9}$ \\
\hline $\mathrm{C}_{1}$ & $2 \ell+2 \sigma$ & $3 \ell+4 \sigma$ & $4 \ell+6 \sigma$ & $5 \ell+8 \sigma$ & $6 \ell+10 \sigma$ & $7 \ell+12 \sigma$ & $8 \ell+14 \sigma$ & $9 \ell+16 \sigma$ \\
\hline $\mathrm{C}_{2}$ & $\mathrm{x}$ & $3 \ell+4 \sigma$ & $\mathrm{x}$ & $5 \ell+8 \sigma$ & $\mathrm{x}$ & $7 \ell+12 \sigma$ & $\mathrm{x}$ & $9 \ell+16 \sigma$ \\
\hline $\mathrm{C}_{3}$ & $\mathrm{x}$ & $\mathrm{x}$ & $4 \ell+6 \sigma$ & $\mathrm{x}$ & $\mathrm{x}$ & $7 \ell+12 \sigma$ & $\mathrm{x}$ & $\mathrm{x}$ \\
\hline $\mathrm{C}_{4}$ & $\mathrm{x}$ & $\mathrm{x}$ & $\mathrm{x}$ & $5 \ell+8 \sigma$ & $\mathrm{x}$ & $\mathrm{x}$ & $\mathrm{x}$ & $9 \ell+16 \sigma$ \\
\hline $\mathrm{C}_{6}$ & $\mathrm{x}$ & $\mathrm{x}$ & $\mathrm{x}$ & $\mathrm{x}$ & $\mathrm{x}$ & $7 \ell+12 \sigma$ & $\mathrm{x}$ & $\mathrm{x}$ \\
\hline
\end{tabular}

Table S8 | Angular momentum states of harmonic generation waves with same circular polarization as that of the fundamental wave $(-\sigma, \ell+2 \sigma)_{\omega}$, and further undergo a spinorbit cascade process. Here the allowed states are $[\sigma, n \ell+2(n-1) \sigma]_{n \omega}$, and ' $\mathrm{x}$ ' means the related harmonic generation is forbidden. This table follows Table S4. 